\title{
Enhanced Deterministic Performance of Panels Using Stochastic Variations of Geometry and Material
}

\author{
Sander van den Broek* \\ Leibniz University Hannover, 30167 Hannover, Germany \\ Sergio Minera ${ }^{\dagger}$, Alberto Pirrera ${ }^{\ddagger}$ \\ University of Bristol, Bristol, United Kingdom, BS8 ITR \\ Paul M. Weaver ${ }^{\S}$ \\ University of Bristol, Bristol, United Kingdom, BS8 ITR \\ University of Limerick, Limerick, Ireland \\ Eelco Jansen ${ }^{\mathbb{I l}}$ and Raimund Rolfes" \\ Leibniz University Hannover, 30167 Hannover, Germany
}

The effect of stochastic variation in material and geometric properties on structural performance is important for robust design. Knowledge of such effects can be acquired by applying variation patterns to a structure using random fields through a Monte Carlo analysis. The output is postprocessed to show the correlation pattern between the stochastic variation of a structural property and a chosen mechanical response measure. The resulting patterns are used to identify areas most susceptible to variations, as well as areas which have the most potential to increase structural performance by varying the material parameter or geometry. By using these maps of local sensitivity to variations with respect to the structural response it is possible to redistribute material properties or geometry to promote certain behavior. This is demonstrated on a flat plate and curved panel, by either varying the Young's modulus, or thickness of the structure to increase the linear buckling load. In both of these variations the average property is set to remain the same as the original structure. Applying the redistribution increased the linear buckling load by up to $29 \%$.

\section{Nomenclature}
$\operatorname{cov}=$ covariance operator
$\mathbf{c}_{i}=$ Coordinates of element $i$

\footnotetext{
*PhD student \& Research Associate, Institute of Structural Analysis, Appelstrasse 9A

${ }^{\dagger} \mathrm{PhD}$ student, Bristol Composites Institute (ACCIS)

Lecturer \& EPSRC Research Fellow, Bristol Composites Institute (ACCIS)

${ }^{\S}$ Professor, Bristol Composites Institute (ACCIS) and Bernal Institute, School of Engineering

"ISenior Faculty member, Institute of Structural Analysis, Appelstrasse 9A

"Professor, Institute of Structural Analysis, Appelstrasse 9A
} 


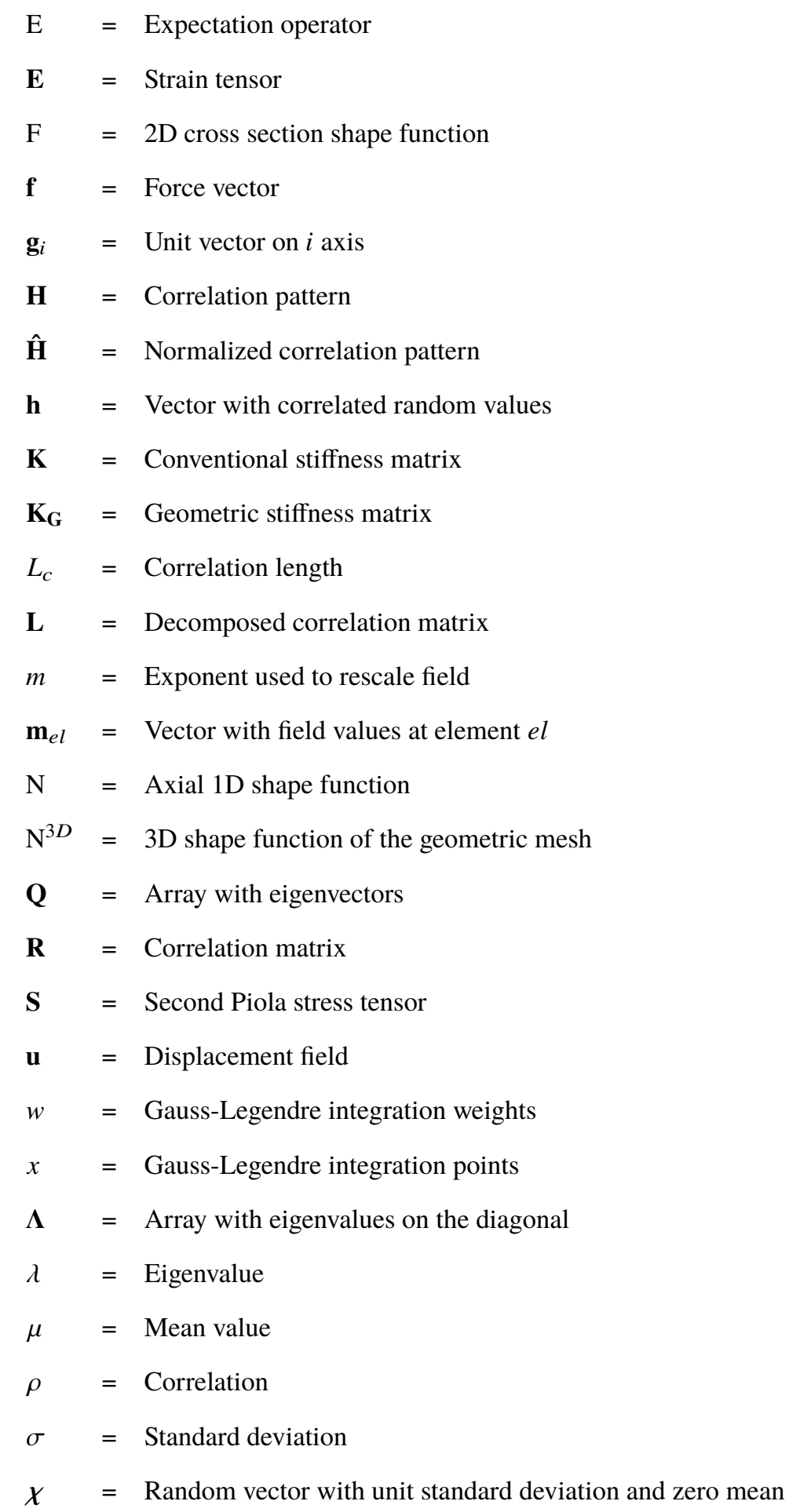

\section{Introduction}

RADITIONALLY structures are designed using constant, meaning uniform and deterministic, material properties throughout the structure and nominal geometry. This representation is not necessarily appropriate, as internal 
stresses, variations in production processes and chemical composition can cause mechanical properties and geometry to vary stochastically.

These variations not only exist within the structural topology, but also between manufactured components thought to be identical. This means that one component coming from the production line may conform to expectations, but another one does not. These variations are usually included in safety factors used within a structural design. Understanding these variations and how they affect performance is a source of extensive research and literature [1-[3].

One of the ways to calculate the effects of stochastic variations is by applying them through random fields [4]. In this way a parameter can vary throughout a structure and, by running sufficient analyses, the effects of the variation can be quantified. Previous uses of this approach have looked at how geometric or material variations can affect mechanical behavior [5- -9].

These analyses usually make assumptions on the spatial distribution of statistical properties, or analyze a range of correlation lengths: The actual distribution depends on the specific structure, manufacturing processes and environmental conditions. Limited information exists in the public domain, with only a few recent papers attempting to find spatial and statistical distributions [10-13]. The approaches discussed in these papers use coupon tests to quantify the variability of mechanical properties and their distributions. This is a costly and time consuming process, which may be affected by factors such as curing temperature, raw material batches or even the technicians making the structure. A potential future alternative is to use non destructive testing to find variations, within composites this could be used to analyze variations in fiber angle, void content as well as other parameters that may affect material properties. This topic is an active field of research [14, 15] which could potentially be used to gain statistical information about structures efficiently and cost effectively.

Variations of nominal material properties and geometry are sometimes introduced purposefully by changing material parameters spatially. One way of achieving local variations is by varying the fiber angle in composites, creating variable stiffness composites [16-18]. Materials can also have other spatially varying material properties, these are often referred to as functionally graded materials. These materials can be used to improve thermal properties [19] or improve the mechanical performance of a structure. This increase can also be achieved by adding variations in the geometry, thereby tailoring the response [20-22]. One example of such an improvement is where the the buckling load of the structure can be increased. Solutions are usually based on a combination of linear buckling modes of the structure. Another approach is to locally vary the thickness, either empirically [23], using periodic functions [24] or by using arbitrary stepwise variations [25]. Some papers directly vary the stiffness by varying the Young's modulus [18, 26]. A few papers combine multiple parameters [27, 28]. There has been some research in optimizing the stiffness topology [26, 28, 29]. These approaches lead to (local) optima, but do not always give physical insight into the physical changes or evolution of the structure. This is possible because the topology is directly coupled with the average sensitivity to variations of

\footnotetext{
*The correlation length, defined in section III.B can be thought of as a characteristic length scale within a random field.
} 
the structure to its response. The changes made to the structure affect the stresses and strains and other mechanical parameters. It is therefore likely possible to make further improvements through an iterative approach. An iterative approach would reveal the non-linear topological change of sensitivity between iterations.

The difference between stochastic approaches and traditional optimization methods is that the results take some degree of random variations into account. The patterns therefore should lead to a design which is less susceptible to deviations of the improved design. This process is different from traditional structural optimization techniques, in which the optimized design becomes increasingly sensitive to deviations from the optimized shape (as optima often lie on a Pareto front of design constraints). Robust optimization is a field of research which is becoming more prominent as engineers realize that uncertainties can have an ever increasing effect on structural performance [30-32].

The work presented herein tries to identify where in the topology variations cause the most influence on a measure of the structural response, and can be considered a more generalized stochastic version of the approach presented by Minera et al. [33] to improve linear buckling loads. Within the current approach a measure, which can be buckling load, stress, displacement or any other output from an analysis targeted to be improved. Property enhancement is achieved by analyzing the effects of fictitious local variations on the structure. This is done by looking at the correlation between these variations and its effect on the structural response. Calculating this correlation at points throughout a structure generates a pattern, which can be used to redistribute the parameter within the structure. This distribution can improve the understanding of the underlying structural mechanics. This knowledge can be used to improve the performance of the structure and identify areas most critical for inspection during manufacture. This approach could potentially be used in additive manufacturing, where there are fewer design constraints and potentially more variation in material properties due to the printing process. Additive manufacturing also makes it possible to vary the composition of the printing material, tailoring it to specific properties needed locally within a structure.

The patterns related to a structural measure are generated through Monte Carlo analyses. These are run using spatially random patterns of fictitious variations and then correlating how the patterns affect the mechanical response, e.g. buckling load, displacements, stresses etc. The variations are analyzed on a flat plate and curved panel, by either varying the Young's modulus within a structure, or by changing the thickness locally. The variations used in this procedure are not related to actual variations in the structure, but are instead fictitious small variations which are purely used to generate the correlation pattern. The correlation pattern is used in turn to tailor the material properties or geometry of the structure to improve performance, as could be done using additive materials or additive manufacturing techniques. These techniques make it possible to theoretically vary the composition of the printing material locally, thereby changing its material properties. The printing process also allows designers to vary geometry such as thickness throughout the structure.

The remainder of this paper first introduces the methods used in section [II] starting with the structural model in section [II.A Section [III.B describes how random fields are generated, how they are mapped is described in section III.C 
The procedure to apply thickness imperfections is explained in section III.D. Section III.Especifies how correlation patterns are calculated, section III.F discusses how they are applied to the structure. Results are shown in section IV. starting with the flat plate example in section IV.A. and continuing with a curved panel example in section IV.B. The paper finishes with a discussion of the results and method as well as some conclusions in section $\mathrm{V}$.

\section{Method}

\section{A. Finite element formulation}

The model used for the structural calculations is based on the Unified Formulation [34]. The implementation used is based on Serendipity Lagrange (SL) elements [35]. These elements utilize SL expansion functions in two dimensions $F(x, z)$, and Lagrange shape functions in the axial direction $(N(y)$ of a 3D element. The implementation is developed for 3D structures with beam-like geometry, in which a cross section is extended axially. This can be done by warping the cross section in the axial direction (to include curvature or tapering), but requires element connectivity to remain the same [36].

The focus of this work is on linear buckling problems, which includes determining the geometric stiffness matrix $K_{G}$. This matrix captures the effect that in-plane forces have on the (out-of-plane) stiffness of the structure, which is essential in buckling problems.

\section{Basic formulation}

The structural model consists of three-dimensional elements. Different shape functions are used in the cross sectional $F(x, z)$ and axial direction $N(y)$ to approximate the displacement field.

Starting with a displacement field $\boldsymbol{u}=[u, v, w]^{\mathrm{T}}$ the Green-Lagrange strain tensor $\mathbf{E}$ can be defined linearly as

$$
E_{i j}=\frac{1}{2}\left(\boldsymbol{u}_{i} \cdot \boldsymbol{g}_{j}+\boldsymbol{u},,_{j} \cdot \boldsymbol{g}_{i}\right)
$$

where commas denote derivatives and $\boldsymbol{g}_{\boldsymbol{i}}$ denotes a unit vector on the $i$ axis. The displacement field $\boldsymbol{u}$ is approximated within the Unified Formulation as

$$
\boldsymbol{u}_{(\mathrm{e})}(x, y, z)=F(x, z) N(y) \boldsymbol{u}_{i}, \quad \text { with } \quad i=1, \ldots, N,
$$

where $N$ are the degrees of freedom of the model. For quasi-static problems the elastic equilibrium is

$$
\delta W_{\mathrm{int}}=\delta W_{\mathrm{ext}},
$$

where $W_{\text {ext }}$ and $W_{\text {int }}$ are the external work and internal energy. Noting that the internal energy of the structure can be 
calculated as the sum of internal energy of all the elements $W_{\text {int }}=\sum_{e} W_{\text {int }}^{(\mathrm{e})}$ the internal energy can be expressed using the stress and strain tensors

$$
\delta W_{\text {int }}^{(\mathrm{e})}=\int_{V_{(\mathrm{e})}} \delta \boldsymbol{E} \cdot \mathbf{S} d V
$$

where $\mathbf{S}$ is the second Piola stress tensor. This can be written as

$$
\delta W_{\text {int }}^{(\mathrm{e})}=\delta \mathbf{u}_{j s}^{T} \mathbf{k}_{(e)}^{i j \tau s} \mathbf{u}_{i \tau}^{T},
$$

where $i, j=1, \ldots, N_{e}, \tau, s=1, \ldots, M, N_{e}$ are the number of Lagrange nodes in the axial direction and $M$ the number of terms in the cross section expansion [35, eq. 11]. The term $\mathbf{k}_{(e)}^{i j \tau s}$ is referred to as the fundamental nucleus, the explicit form of which can be found in [34, 37]. The fundamental nuclei can be assembled into a global stiffness matrix $\mathbf{K}$ in the form

$$
\mathbf{K u}=\mathbf{f},
$$

which can be solved to find generalized displacements.

In order to calculate the linear buckling load an additional geometric stiffness matrix $\mathbf{K}_{G}$ is needed. For the sake of brevity the full form is omitted, but can be found in [33, 38, 39]. Linear buckling can be derived as an eigenvalue problem in the form [40, ch. 18]

$$
\left(\mathbf{K}+\lambda_{c r} \mathbf{K}_{G}\right) \delta \mathbf{u}=\mathbf{0},
$$

where $\delta \mathbf{u}$ represents a buckling mode with an indeterminate amplitude. The scalar value $\lambda$ is a proportionality factor which relates applied force $\mathbf{f}$ and the stresses, which are used to generate the geometric stiffness matrix. The buckling load can be solved as the non-trivial solution to

$$
\operatorname{det}\left(\mathbf{K}+\lambda_{c r} \mathbf{K}_{G}\right)=0
$$

whereby solving for $\lambda_{c r}$ and subsequently identifying the load factor as

$$
\mathbf{f}_{c r}=\lambda_{c r} \mathbf{f}
$$

where $\mathbf{f}_{c r}$ is the buckling load.

\section{Geometric representation}

The element stiffness matrix is obtained by integrating the terms of equation (5) throughout the element domain. When modelling complex geometries the correct geometrical description is of fundamental importance. The SL 


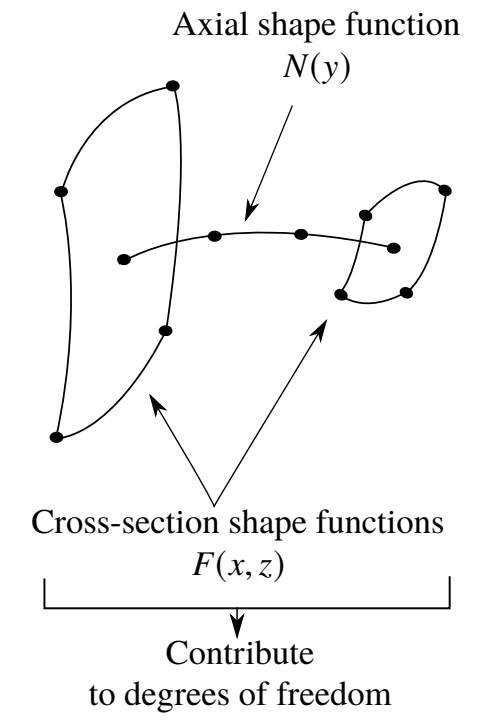

(a) SL element shape function nodes

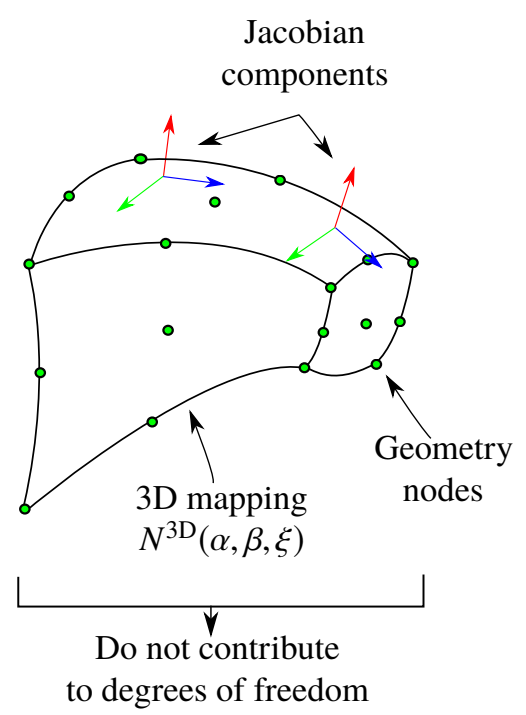

(b) Geometric respresentation $N^{3 D}$ nodes

Fig. 1 Element discretization and shape functions, [36].

expansion functions defined in Section IIII.A are used to enrich the kinematics in the cross-section. These functions are integrated over the cross section of the structure, which requires a transformation of coordinates. If the edges of a quadrilateral element are straight, the approximation of the geometry is obtained through linear mapping by using linear Lagrange polynomials, which in this case coincide with SL functions of order 1. This approach does not allow for an accurate representation of curved geometries. When using isogeometric formulations the same functions describe the displacement field and the geometry. The advantage of this approach is that modeling the geometry correctly, automatically improves the accuracy of the field. This may however come with a high computational cost, especially when Unified Formulation is used. An alternative approach is to discretize the geometry and structure separately, as is done in the current approach. This approach uses higher order meshes for the geometry, and higher order Lagrange shape functions for coordinate transformations.

The hierarchical nature of the SL shape functions makes it possible to use them within curved elements, as the expansion order can be increased as needed. The approach adopted in the current structural model aims to do this without adding to the degrees of freedom of the structure. This can be done by blending the structure with higher order polynomials [41], which can become cumbersome for distorted or large meshes. Another approach is to use exact geometric descriptions through non-linear functions [42, ch 5]. This approach requires an exact geometric description to be given analytically, limiting the geometry that can can be analyzed. The approach used in the current model is non-isoparametric, utilizing higher order Lagrange shape functions to describe geometry.

The current approach uses a higher order mesh generated using gmsh [43], generating a mesh with elements of 9 or 16 nodes in the cross section. This gives a $2 \mathrm{D}$ shape function $N^{2 D}(\alpha, \beta)$ to describe the geometry of a cross section 
which is independent of the structural shape functions $F(x, z)$ and $N(y)$. The function $N^{2 D}(\alpha, \beta)$ is defined in the range $[-1,1]^{2}$ and defines the mapping between the global coordinates and local element coordinates

$$
\boldsymbol{x}=N_{k}^{2 \mathrm{D}}(\alpha, \beta) \boldsymbol{x}_{k},
$$

where $\boldsymbol{x}_{k} \in \mathbb{R}^{2}$ are the position vectors of the nodes of the element, and $k=1, \ldots, N_{n e}$, with $N_{n e}$ the number of nodes; 9 or 16 node Lagrange elements are supported by the current implementation.

To model varying thickness this approach is extended by changing the shape function of the cross section to vary at every integration point. This adds another dimension to the shape function $N^{2 D}(\alpha, \beta)$ to account for the change in the axial direction. This new function $N^{3 D}(\alpha, \beta, \xi)$ can change the cross section mapping as a function of $\xi$, making it possible to model tapered structures.

The number of nodes used to describe the geometry $\left(N^{3 D}(\alpha, \beta, \xi)\right.$ is independent from the number of degrees of freedom in the structural model $(F(x, z)$ and $N(y))$. The degrees of freedom of the structural and geometric mesh are shown in fig. 1. The 2D shape function $N^{2 D}$ is identical to $N^{3 D}$ in structures which are prismatic. For non-prismatic structures this shape function is calculated at every integration point in the $\xi$ direction.

The current implementation uses 3D geometric Lagrange shape functions to take thickness variations into account efficiently. This geometric mesh overlaps the structural mesh, but uses higher order brick elements of 27 or 64 nodes. These shape functions reduce the amount of computation needed during assembly. The 3D geometric mesh shape functions are used in section III.C and section III.D to map variations to the structure, and to calculate the volume and average material properties of the structure.

\section{B. Random field generation}

Random fields, also known as stochastic fields, are fields in $n$-dimensional space which spread a parameter in space with a distribution. Values within a field are correlated with each other. There are many methods which can be used to generate random fields [4]. These methods have their own advantages and disadvantages. The technique used here is Covariance Matrix Decomposition (CMD) [44, 45, sec. 5.3.2]. This choice is made due to the relative ease of implementing customized correlation functions. CMD creates fields directly from an autocorrelation matrix by decomposing it and multiplying the decomposed array with a random vector. The values of a random field are usually generated from a $(\log )$ normal distribution with an associated mean value $(\mu)$ and standard deviation $(\sigma)$. Actual variations may not be in such a distribution, but are often assumed to be such as a simplification, or due to a lack of experimental data. These points are correlated in space, so giving a relationship to the value of a coordinate and those in 
its vicinity. The mathematical definition [46, ch. 10] of correlation between points $i$ and $j$ of field $\mathbf{h}$ is

$$
\rho_{h_{i}, h_{j}}=\frac{\operatorname{cov}\left(h_{i}, h_{j}\right)}{\sigma_{i} \sigma_{j}}=\frac{\mathrm{E}\left[\left(h_{i}-\mu_{i}\right)\left(h_{j}-\mu_{j}\right)\right]}{\sigma_{i} \sigma_{j}}
$$

where $\mathrm{E}$ is the expectation operator used in probability and equals the mean value of a distribution given an infinite amount of samples. Equation (11) can be used to calculate the autocorrelation between points, to generate fields these correlations have to be given as an input. The most common functions found in literature are based on an exponential function. The correlation function used to generate the fields here is

$$
\rho_{s, \exp }=e^{-\left(\frac{\Delta L}{L_{c}}\right)^{2}}
$$

in which $L_{c}$ is the correlation length, and $\Delta L$ the distance between two points. This relationship assumes stationarity, as the field is only dependent on distance, and does not change throughout the structure. The term $\Delta L$ traditionally refers to Euclidean distance, but as we are concerned with thin-walled structures, the geodesic length is used instead. Using geodesic lengths creates a more accurate measure for generating random fields than Euclidian distance, particularly for highly curved structures [47]. Structures which are relatively flat might have a bottom and top which are close to each other in a Euclidian sense, but distant in a geodesic sense. The geodesic lengths of the current implementation are calculated using the heat method of Crane et al. [48], using normalized heat gradients to calculate the shortest path through solving the Poisson's equation. This can be done with limited computational costs, as these turn out to be linear problems. The random field method used in this paper does not offer a continuous function and instead discretizes points into space, which are then coupled to points in the structure. The space between random field nodes should be between $\frac{L_{c}}{4}$ to $\frac{L_{c}}{2}$ for the correlation function used [49]. In the context of this paper the correlation length represents a measure of the resolution of the correlated structure. A shorter correlation length allows variations to be more local, allowing patterns to be more localized, at the cost of taking longer to converge. Having a large correlation length therefore makes it harder for smaller details to converge, large scale variations make it easier to converge to a pattern.

Using CMD, random fields are calculated through multiplication of a matrix and a random vector

$$
\mathbf{h}=\mathbf{L} \chi
$$

in which $\mathbf{L}$ is a decomposed version of the correlation matrix $\mathbf{R}$ and $\mathcal{\chi}$, a vector with random entries of zero mean and unit variance. This decomposition has to be done in such a way as to generate a correlated vector $\mathbf{h}$ with a mean of zero and unit variance. 
The first step in generating random fields is to build a correlation matrix of all vertices of the field

$$
R_{i j}=\frac{\operatorname{cov}\left(h_{i}, h_{j}\right)}{\sigma_{i} \sigma_{j}} \rightarrow \mathbf{R}=\left[\begin{array}{cccc}
1 & \rho\left(h_{1}, h_{2}\right) & \ldots & \rho\left(h_{1}, h_{n}\right) \\
\rho\left(h_{2}, h_{1}\right) & 1 & \ldots & \rho\left(h_{2}, h_{n}\right) \\
\vdots & & \ddots & \vdots \\
\rho\left(h_{n}, h_{1}\right) & \rho\left(h_{n}, h_{2}\right) & \ldots & \rho\left(f_{n}, f_{n}\right)
\end{array}\right]
$$

in which $\rho\left(h_{i}, h_{j}\right)=\rho\left(h_{j}, h_{i}\right)$, noting that the correlation here can be calculated using eq. 12.

Taking the definition of covariance

$$
\operatorname{cov}\left[h_{i}, h_{j}\right]=\mathrm{E}\left[h_{i} h_{j}\right]-\mathrm{E}\left[h_{i}\right] \mathrm{E}\left[h_{j}\right]
$$

and keeping in mind the field has a zero mean, it is possible to show that $\mathbf{R}$ can be decomposed into two matrices

$$
\begin{aligned}
\mathbf{R} & =\operatorname{cov}[\mathbf{h}, \mathbf{h}]=\mathrm{E}\left(\mathbf{h}, \mathbf{h}^{T}\right)-0 \cdot 0 \\
& =\mathrm{E}\left(\mathbf{L} \chi(\mathbf{L} \chi)^{T}\right)=\mathbf{L E}\left(\chi \chi^{T}\right) \mathbf{L}^{T}=\mathbf{L} \mathbf{I} \mathbf{L}^{T}=\mathbf{L} \mathbf{L}^{T},
\end{aligned}
$$

where $\mathbf{I}$ is an identity matrix. Noting that from eqs. (12) and 14) the matrix $\mathbf{R}$ is symmetric and positive semi-definite, the eigenvalues should not be negative. This expression exploits the independence of the components of $\chi$. Decomposing is done by using eigendecomposition in the form of

$$
\mathbf{R}=\mathbf{Q} \mathbf{\Lambda} \mathbf{Q}
$$

where $\boldsymbol{\Lambda}$ is a diagonal matrix with the eigenvalues of $\mathbf{R}$ on the diagonal, and $\mathbf{Q}$ contains the eigenvectors of the matrix. The matrix $\mathbf{L}$ can be extracted as

$$
\mathbf{R}=\mathbf{Q} \hat{\mathbf{\Lambda}} \hat{\mathbf{\Lambda}} \mathbf{Q}=\mathbf{L} \mathbf{L}^{T} \rightarrow \mathbf{L}=\mathbf{Q} \hat{\mathbf{\Lambda}},
$$

in which $\hat{\mathbf{\Lambda}}=\operatorname{diag}(\sqrt{\lambda})$, where $\lambda$ are the eigenvalues of the $\mathbf{R}$ matrix. Using the decomposed correlation matrix $\mathbf{L}$ it is possible to generate random fields using equation eq. [13, , requiring minimal additional computational costs.

\section{Random field mapping to structure}

The random fields in the current implementation are generated on a surface, while the structural model is based on $3 \mathrm{D}$ elements. In order to assign material properties to each integration point using the values of the random field it is 
necessary to implement a mapping procedure.

The mapping procedure used within this paper separates the random field mesh and the structural mesh. This is done so that in other applications the geodesic length can be calculated with a greater accuracy, being able to more accurately represent the curved geometry. It also makes it possible to refine the structure without at the same time refining the random field discretization.

By using the higher order shape functions of the geometric mesh (section III.A.2) it is possible to include the curvature within an element in the mapping procedure. The mapping procedure starts by projecting each node of the geometric mesh to the random field surface, finding the shortest Euclidian distance. This only has to be done once, which saves time during Monte Carlo analyses. While initializing each analysis the values of the random field are calculated for every geometric node using the four node shape function of the random field element.

During assembly, material properties of each integration point are interpolated using the shape function of the geometric mesh. This process has the advantage that by using higher order elements (e.g. 27 nodes) it is possible to have a non-linear variation of properties within an element. It is therefore possible to represent the structure with larger elements and still have an accurate material property distribution. As a consequence the mapping procedure can have a higher fidelity than would be possible with the linear shape functions of the 8 node structural elements. The optimal discretization of the random field is therefore also dependent on the order of the geometric Lagrange elements, and the structural SL elements in addition to the correlation function used.

The illustration in fig. 2 shows the mapping process, where at an integration point the coordinates within the element coordinate system are used to interpolate values of the geometric mesh, or mathematically as

$$
h_{i}=\mathbf{m}_{e l} \mathbf{N}_{e l}^{3 D}\left(\alpha_{i}, \beta_{i}, \xi_{i}\right)
$$

where $h_{i}$ is the field value at a point $i, \mathbf{m}_{e l}$ a vector with the field values at element $e l$, and $\mathbf{N}_{e l}^{3 D}\left(\alpha_{i}, \beta_{i}, \xi_{i}\right)$ the shape function evaluated at element coordinate $i$ with components $\alpha, \beta, \xi$. It should be noted that this approach has a constant value assumption through thickness, which is why the value is the same for $\beta$ between $[-1,1]$.

Random field values are normalized to zero mean and unit variance. Applying the field to a variation in the Young's modulus would require the addition of the mean and multiplication with the standard deviation as

$$
E_{i}=E_{\mu}+h_{i} E_{\sigma}
$$

in which $E_{i}$ is the Young's modulus at point $i, E_{\mu}$ is the mean value of $E, h_{i}$ is the value of the random field at the point and $E_{\sigma}$ is the standard deviation of the Young's modulus. 


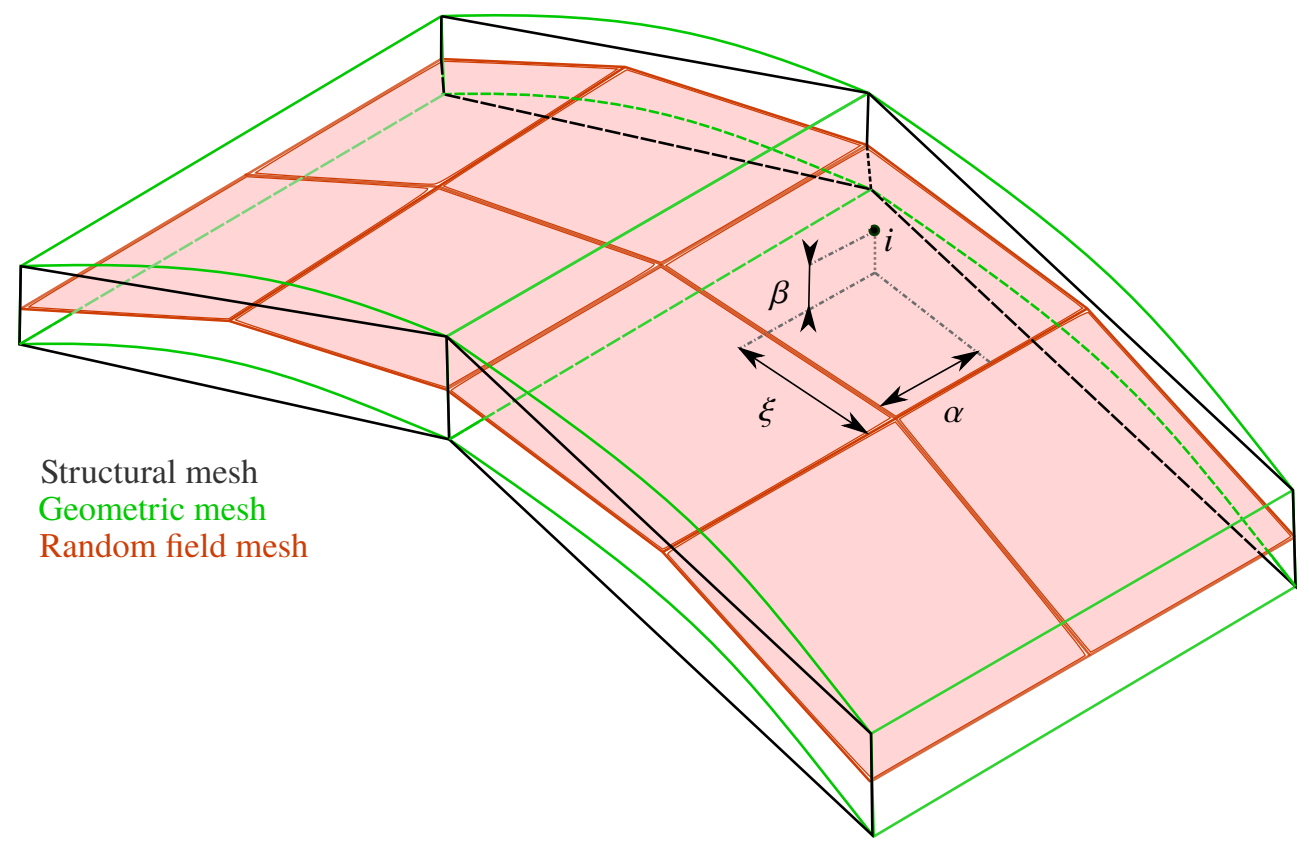

Fig. 2 Separate discretization and mapping procedure between meshes, and coordinates of point $i$.

\section{Applying thickness imperfections}

Applying variations to the thickness in the structure requires changing the 3D mesh to reflect the new geometry. This is done by mapping the random field to perturbations to the structural mesh.

\section{Perturbing the mesh}

In section III.C a mapping between the random field and nodes of the geometric mesh $\left(N^{3 D}\right)$ is described. This process gives interpolated scalar values of the random field to every node in the geometric mesh. This scalar value is used to determine the amount of displacement that should be applied to the node.

The approach used in the current work determines the amount of displacement of a coordinate by scaling the displacement with the distance from the random field surface. This approach makes it possible to apply symmetric thickness variations, by having the random field in the middle of the structural mesh. It also makes it possible to vary in one direction, by having the random field intersect the surface which should not be varied.

The direction in which the node is displaced is taken from the derivative of the shape function with respect to the element normal direction. In the convention used within this paper this gives the normal direction relative to the surface of the structure. An unrotated element has its $\alpha, \beta, \xi$ components aligned with $x, z, y$, with the normal being defined as the component in $z$. With the convention used the normal direction therefore equal to $\mathbf{g}_{\beta}=\mathbf{x} \frac{\partial N^{3 D}}{\partial \beta}$, where $\mathbf{g}_{\beta}$ is a unit length normal vector of a coordinate in the normal $(\beta)$, and $\mathbf{x}$ an array of the physical coordinates of an element. This solution may not be unique, if a node is used in multiple elements. In those cases the average normal direction of all elements containing the node is used. The distance $d$ between a physical node and the random field surface is calculated 
numerically. The displacement is applied to node $i$ by adding a perturbment to the coordinates of the nodes

$$
\mathbf{c}_{i, \text { new }}=\mathbf{c}_{i, \text { old }}+\delta \mathbf{d}_{i}=\mathbf{c}_{i, \text { old }}+d_{i} \sigma_{t} \mathbf{g}_{\beta} h_{i}
$$

in which $d_{i}$ is the original distance between the node and random field surface and $\mathbf{c}_{i}$ the coordinates of node $i$.

\section{E. Calculating the correlation of a parameter on the structure}

By running an analysis $n$ times it is is possible to calculate how variations of material or geometric parameters affect the structure. This approach makes it possible to identify areas which most influence the mechanical response. In doing so, offering valuable information on the relationship of buckling load to stiffness variation, stress to displacement or any other combination of parameters.

The correlation is calculated at the same points in which the random field is discretized, the points are then evaluated for parameters $a$ and $b$. Using the definition of correlation of eq. (11) the correlation of each point $a$ on the structure is calculated relative to parameter $b$. For the examples discussed in the results section the parameter $a$ would equal the Young's modulus or thickness and the parameter $b$ the first linear buckling load. For this example the pattern can be evaluated as

$$
H_{i}=\frac{\sum_{j=1}^{n}\left(f_{j}-\bar{f}\right)\left(E_{i, j}-\bar{E}\right)}{\sqrt{\sum_{j=1}^{n}\left(f_{j}-\bar{f}\right)^{2}} \sqrt{\sum_{j=1}^{n}\left(E_{i, j}-\bar{E}\right)^{2}}},
$$

where $H_{i}$ is the correlated value at point $i, f_{j}$ the buckling load at run $j, \bar{f}$ the sample mean buckling load over all $n$ runs, $E_{i, j}$ the Young's modulus at point $i$ at run $j$ and $\bar{E}$ the sample mean Young's modulus over all runs.

\section{F. Applying the correlation pattern to improve mechanical response}

The map of correlation gives a direct indication of how the structure responds to a variation of a parameter at a specific location. Changing the base-state to incorporate the variation pattern of parameter $a$ (e.g. Young's modulus) can therefore increase or decrease the parameter $b$ (e.g. buckling load). In order to map this correlation pattern to the structure it is first normalized by fitting it into a range $[0,1]$. Using this normalized pattern it is used to redistribute the parameter with the function

$$
a_{i}=a_{\min }+\left(a_{\max }-a_{\min }\right) \hat{H}_{i}
$$

where $\hat{H}_{i}$ is the value of the normalized correlation pattern at $a_{i}$.

The distribution of $\hat{H}$ is not necessarily symmetric, making it necessary to manipulate this pattern to improve performance or move the sample mean within a selected range. An extension is therefore introduced to eq. 23. to

$$
a_{i}=a_{\min }+\left(a_{\max }-a_{\min }\right) \hat{H}_{i}^{m}
$$


in which either the exponent $m$ or range of $a \in\left[a_{\min }, a_{\max }\right]$ can be calculated to ensure the average value of the property remains unchanged.

In the analyses shown in this paper $a$ is either a Young's modulus (a functionally graded material), or the thickness of the structure, the parameter $b$ is the linear buckling load. In these analyses a large value for $m$ would result in very local increases in the Young's modulus, or thickness. A small value for $m$ would cause localized low values of the distribution. The exponent $m$ and range of the pattern are related. When $m$ is decreased the average value of the pattern increases, while increasing $m$ causes it to decrease. There is a unique value of $m$ for every specified range in which the average value (Young's modulus or thickness) remains the same.

\section{Calculating volume and average properties in an analysis}

When enhancing the structure it is important to analyze the change in mass or average material property within a structure. Increasing the stiffness of a structure by adding mass or increasing the average Young's modulus is trivial. The volume of elements are calculated by using their shape functions, which represents a mapping between coordinate systems. Taking the definition of a volume element

$$
d V=\rho\left(u_{1}, u_{2}, u_{3}\right) d u_{1} d u_{2} d u_{3},
$$

The volume of an element can be found by integrating $d V$ over the volume of an element. Utilizing the 3D Jacobian

$$
J 3 D=\left|\begin{array}{lll}
\frac{\partial N_{1}^{3 D}}{\partial \alpha} & \frac{\partial N_{1}^{3 D}}{\partial \beta} & \frac{\partial N_{1}^{3 D}}{\partial \xi} \\
\frac{\partial N_{2}^{3 D}}{\partial \alpha} & \frac{\partial N_{2}^{3 D}}{\partial \beta} & \frac{\partial N_{2}^{3 D}}{\partial \xi} \\
\frac{\partial N_{3}^{3 D}}{\partial \alpha} & \frac{\partial N_{3}^{3 D}}{\partial \beta} & \frac{\partial N_{3}^{3 D}}{\partial \xi}
\end{array}\right|,
$$

and using Gauss-Legendre quadrature the volume of an element is integrated numerically as

$$
V_{i}=\sum_{i=1}^{N} \sum_{j=1}^{N} \sum_{k=1}^{N} J 3 D\left(x_{i}, x_{j}, x_{k}\right) w_{i} w_{j} w_{k}
$$

where $x_{i, j, k}$ are the integration points in the range $[-1,1], w_{i, j, k}$ integration weights, and $N$ the integration order.

To calculate the average value of the property a weighed integration is done of the volume, which is then divided by the structure's volume. For a property $n$ within a structure this becomes

$$
\mu_{n}=\frac{\sum_{i=1}^{N} \sum_{j=1}^{N} \sum_{k=1}^{N} J 3 D\left(x_{i}, x_{j}, x_{k}\right) p_{n}\left(x_{i}, x_{j}, x_{k}\right) w_{i} w_{j} w_{k}}{\sum_{i=1}^{N} \sum_{j=1}^{N} \sum_{k=1}^{N} J 3 D\left(x_{i}, x_{j}, x_{k}\right) w_{i} w_{j} w_{k}}
$$

where $p_{n}\left(x_{i}, x_{j}, x_{k}\right)$ is the property $n$ at integration point $x_{i}, x_{j}, x_{k}$. 


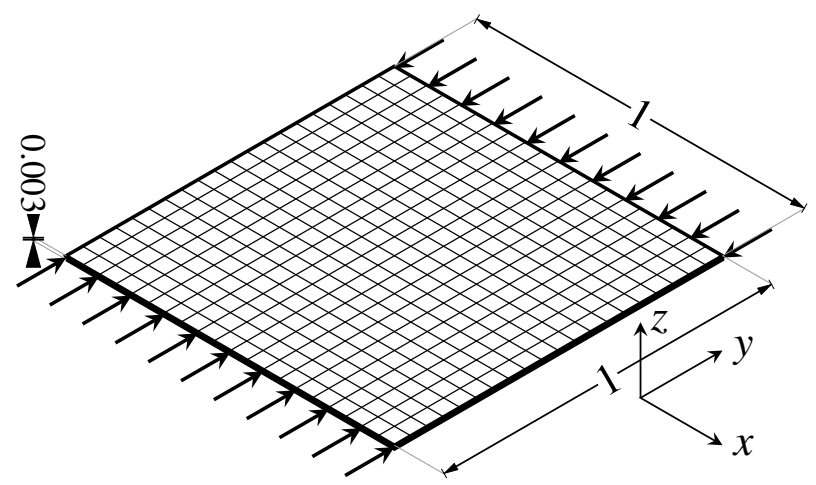

Fig. 3 Rectangular plate, dimensions in $\mathbf{m}$

\section{Results}

The methods discussed in the previous section were applied to two different structures; a square flat plate and a curved panel. Both of these examples analyze variations of the Young's modulus and the thickness separately. These variations are applied intrusively, by perturbing the mesh in the case of thickness variations, and non-intrusively by changing the scalar value of material properties at integration points in the case of Young's modulus variations.

\section{A. Rectangular plate}

The first example consists of a rectangular plate of $1 \times 1 \mathrm{~m}$ and a thickness of $3 \mathrm{~mm}$, as shown in fig. 3 It is modeled using a 20x20 mesh with Serendipity Lagrange 3rd order elements (described in section [III.A). The material properties used are $\mathrm{E}=181 \mathrm{GPa}, v=0.3$. A unit force is applied to the $y=0$ and $y=1$ edges as a uniform distributed load on the surface, with a total force of $1 \mathrm{~N}$. Out-of-plane (z) displacement is restricted on all edges, $x$ displacement is also restricted on the $x=0$ and $x=1$ edges. The linear buckling load is $16892 \mathrm{~N}$, with the buckling mode shown in fig. 4 Prior to generating the correlation pattern the buckling load and mode were verified using the finite element code DIANA [50].

\section{Young's modulus variation}

5000 runs were made in which a random field with a correlation length $L_{c}=0.2 \mathrm{~m}$ was applied to a standard deviation in the Young's modulus of $1 \mathrm{GPa}$. An example of such a distribution can be found in section IV.A.1. The correlation pattern of the Young's modulus to first buckling load among all 5000 runs is shown in fig. 6a These runs are used to generate correlation patterns with which the Young's modulus is redistributed in order to increase the linear buckling load. This could be done by utilizing functionally graded materials, where the stiffness varies throughout the geometry.

This pattern is normalized and used to redistribute the Young's modulus of the plate in the range of 108-254 $\mathrm{GPa}$, which is $\pm 73 \mathrm{GPa}$ the baseline value. This range is an assumption using data of laser deposition range ratios in 


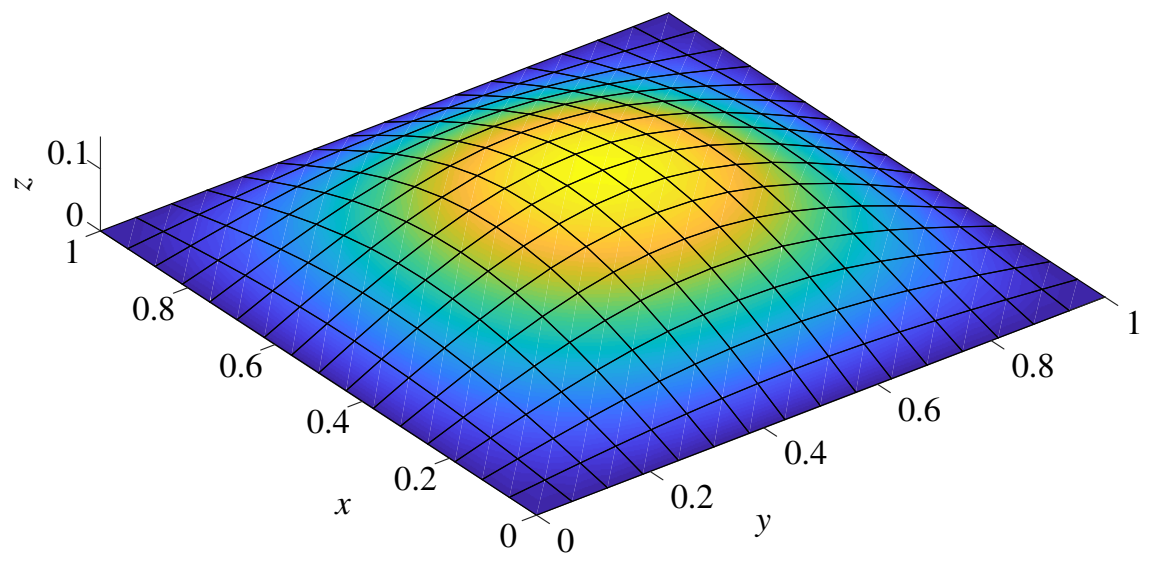

Fig. 4 First buckling mode of the rectangular plate

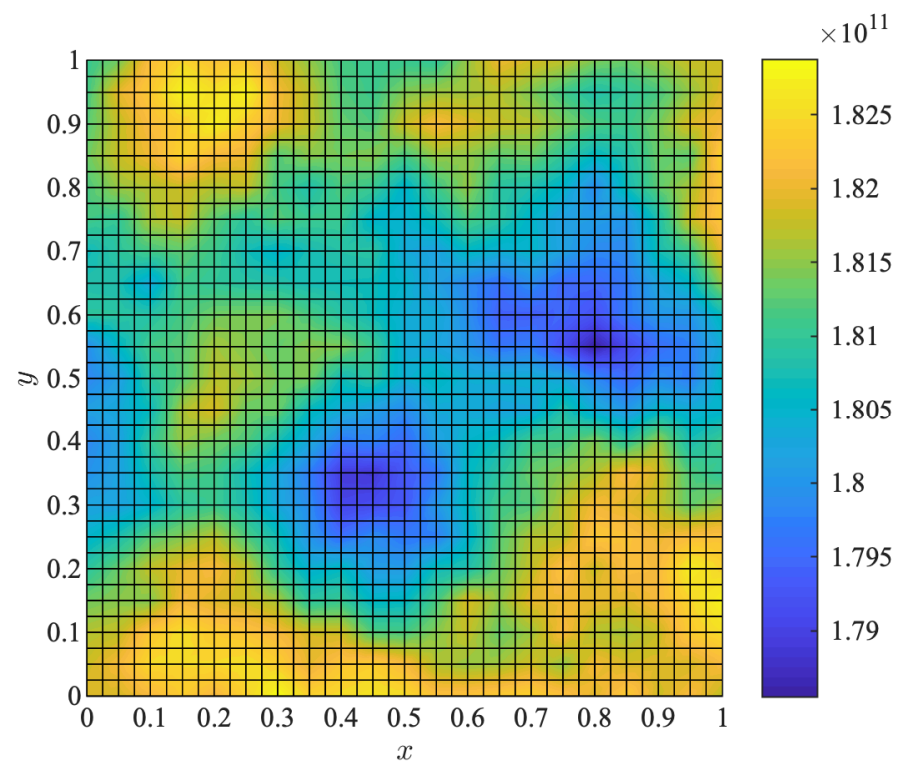

Fig. 5 Example of randomly distributed Young's modulus on the flat plate, Pa 


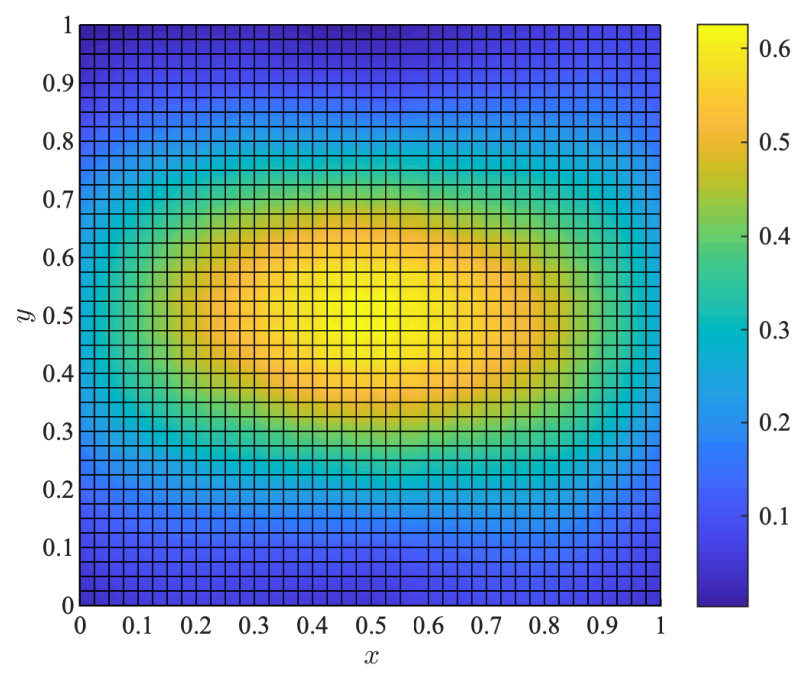

(a) Correlation of $E$ with first linear buckling load

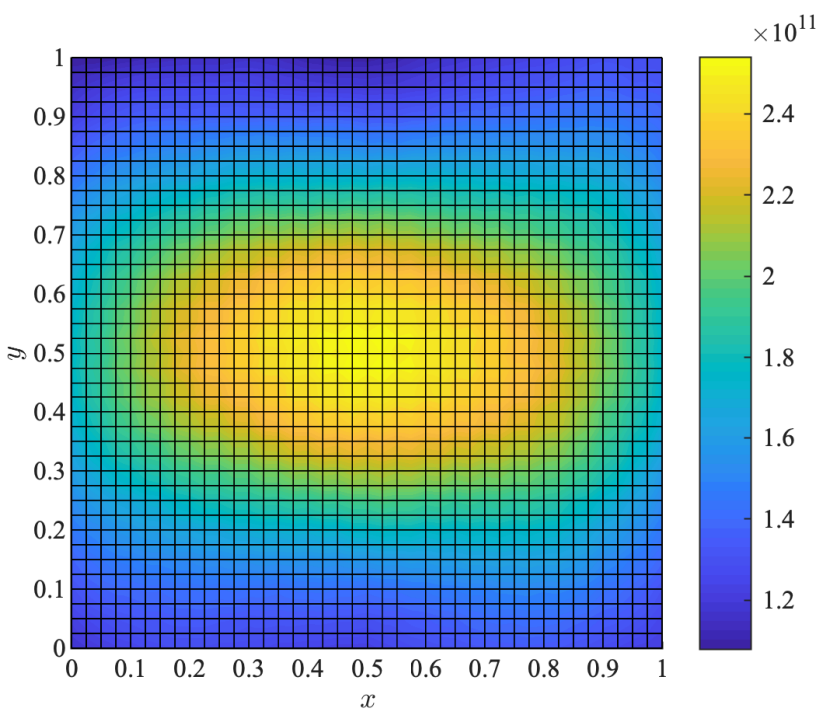

(b) Young's modulus distribution $(m=0.84)$, Pa

Fig. 6 Calculated correlation pattern and applied Young's modulus distribution of flat plate example

Ti6A14V/TiC functionally graded materials achieved by Mahamood and Akinlabi [51]. Unfortunately, this paper does not include mechanical properties, the Young's modulus range is therefore assumed using the deposition ratios achieved. The average Young's modulus of the analyses was kept equal to that of the baseline analysis (181 GPa). By having a fixed range, and a set average in the pattern, it is possible to determine the exponent $m$ in eq. (24) to ensure the average Young's modulus in the plate equals that of the baseline. Repeating this procedure for a variety of property ranges, the results of which are shown in fig. 7. The largest increase was found for the range 108-254 GPa, using a scaling parameter of $m=0.84$. This is a unique value for a specific material range, pattern and average value of the pattern. The associated material distribution can be found in fig. $6 \mathrm{~b}$. applying the same loads and boundary conditions to the plate results in an $8.6 \%$ increase in linear buckling load $(18363 \mathrm{~N})$.

\section{Thickness variation}

Thickness is varied over 5000 samples. The fictitious variations have a standard deviation of $5 \%$ of thickness with a correlation length of $0.2 \mathrm{~m}$. The correlation of the thickness variation with the linear buckling load can be found in fig. $8 \mathrm{a}$ The load vector is updated every step to ensure that the total load magnitude is unit, and distributed proportional to the local thickness. Analyses were run over a range of thickness ranges between $\pm 5 \%$ and $\pm 50 \%$, the results of which can be found in fig. 9 The best result was found using a range of $-50 \%$ to $35 \%$ of the original thickness $(0.0015-0.00405$ $\mathrm{m}$ ), using a scaling coefficient $m=0.7409$ to retain constant mass. In contrast to the Young's modulus example the results here are not the best with the largest range. Using a large range causes the scaling parameter used to retain constant mass be more focused in the material thickening. In this case this would cause a large local increase of bending stiffness while reducing the bending stiffness in other parts of the structure. An optimal is therefore found as a tradeoff 


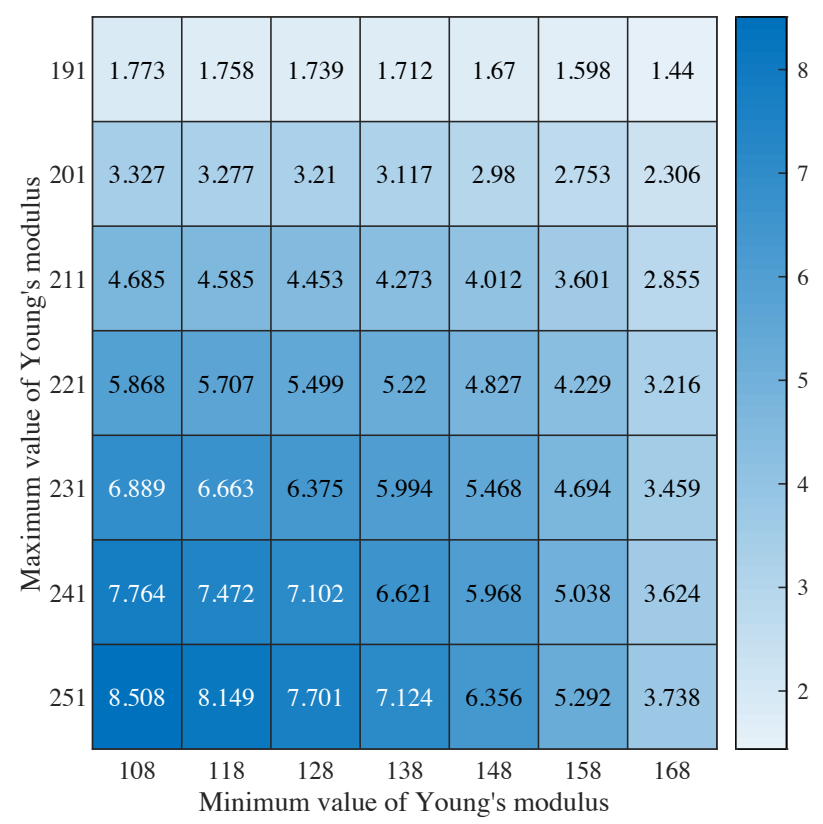

(a) Percentage increase in linear buckling load

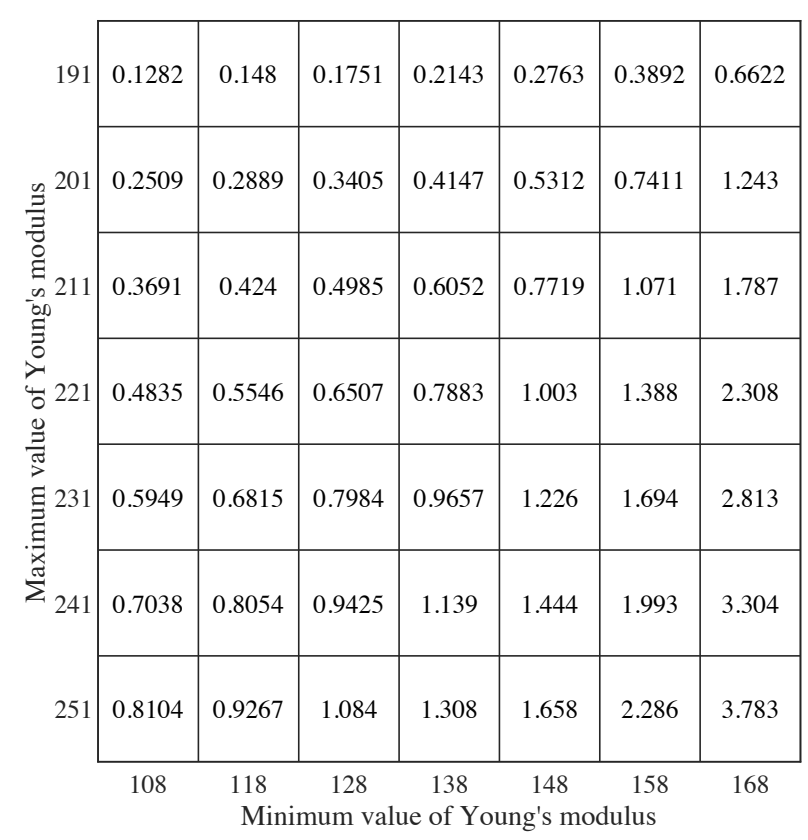

(b) Parameter $m$ (eq. 24) for constant Young's modulus

Fig. 7 Linear buckling load improvement of flat plate with Young's modulus variation, retaining baseline average

between local stiffening and a decrease in stiffness in other areas. It is quite possible that extending the range through sequential iterative steps will provide a better result. Applying this pattern, shown in fig. $8 \mathrm{~b}$ increases the linear buckling load by $16.9 \%$ to $19754 \mathrm{~N}$.

\section{Interpretation}

An interpretation of the results is made using the effect on the prebuckling stress distribution, and the buckling state through buckling mode and strain energy density. The strain energy density gives an indication of the local stresses and strains that are locally stored through elastic deformation. The center of the plate is stiffened in both parameter studies. By examining the the strain energy density with definition $U=\frac{1}{2} \varepsilon_{i j} \sigma_{i j}$ [52, p. 122] shown in fig. 10, it can be deduced that the strain energy density is lower at the center of the plate, where the buckle forms. The reduction is due to a redistribution where the strain energy density is relocated away from the center and towards the loaded edges. The redistribution of strain energy density is much more prominent than the redistribution of prebuckling stresses. The redistribution of the thickness and strain energy cause the buckling mode to change, moving the curvature of the buckled shape from the center towards the edges, as shown in fig. 11 This effect is due to the local increase of stiffness in the center of the plate, which causes the mode shape to change where the curvature is more prominent at the edges. 


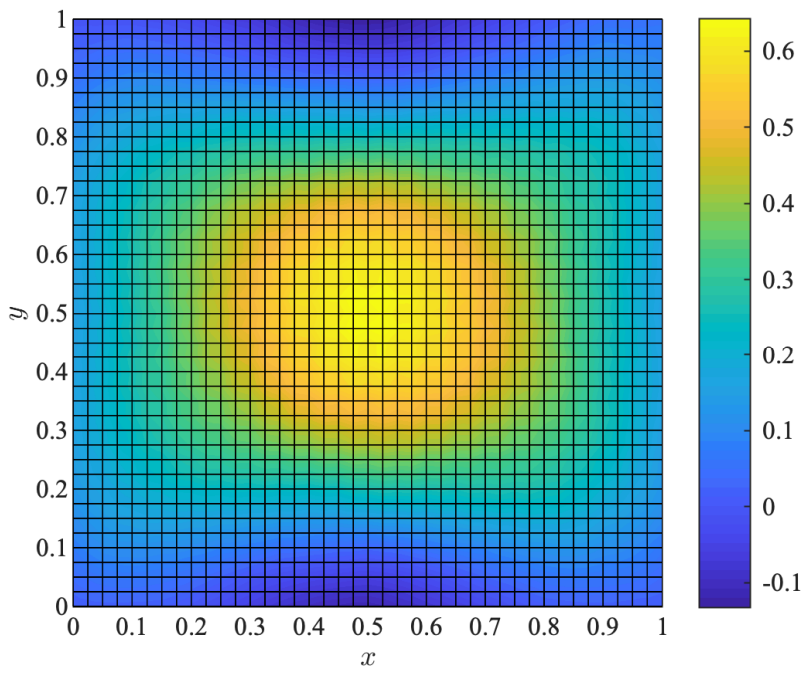

(a) Correlation of panel thickness to linear buckling load

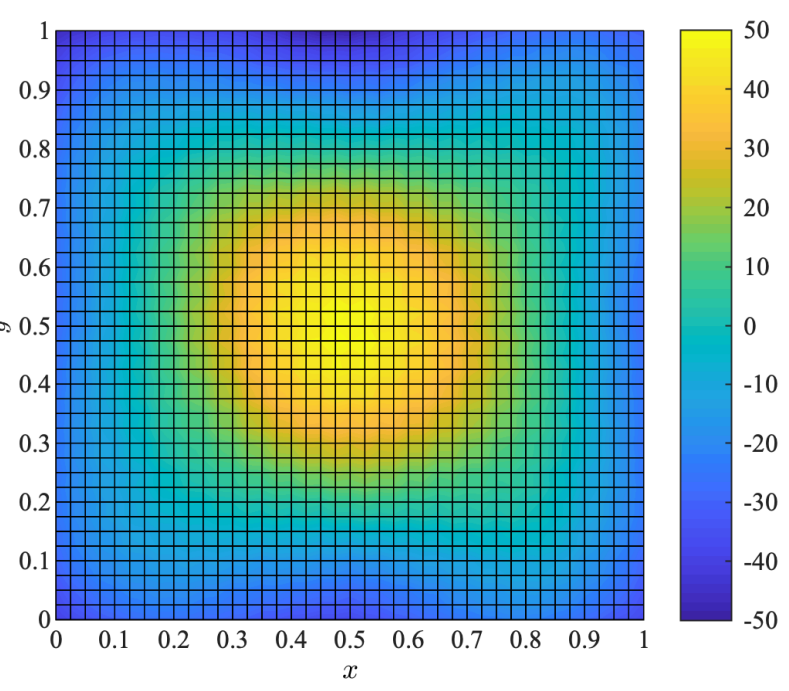

(b) Change of thickness relative to original thickness, \%

Fig. 8 Correlation pattern and redistribution of thickness within the flat plate

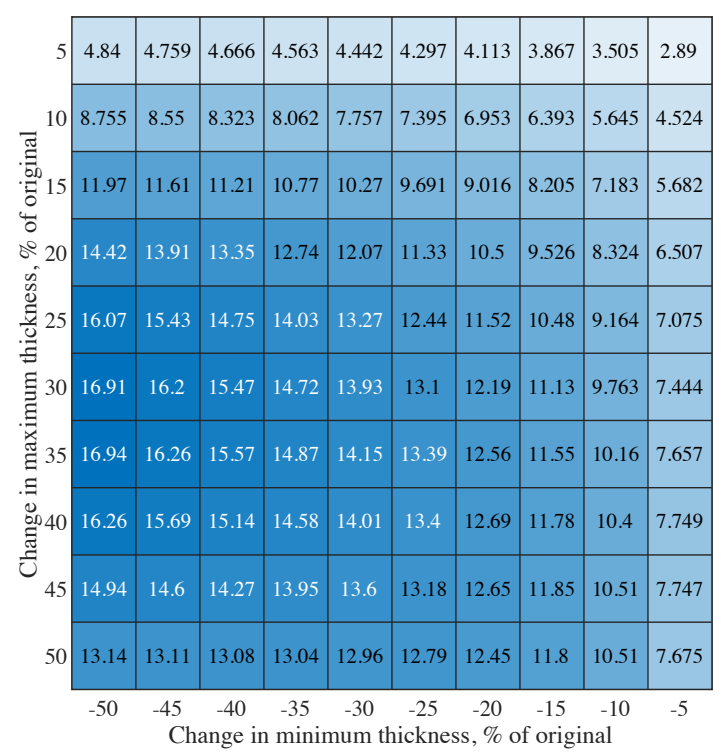

(a) Percentage increase in linear buckling load
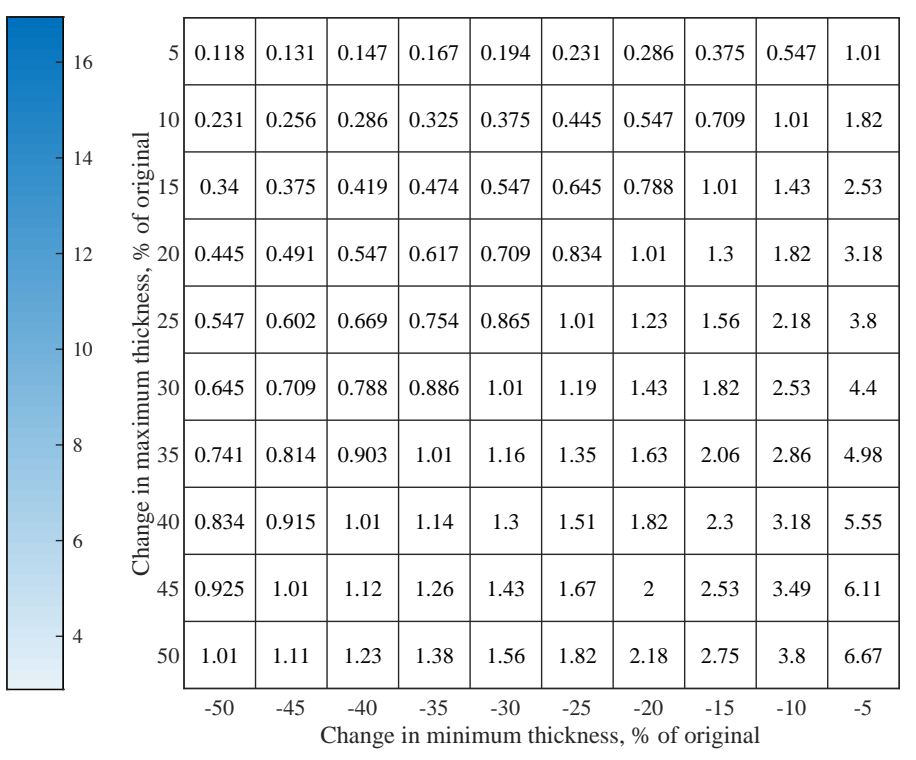

(b) Scaling parameter $m$ (eq. 24) for constant mass

Fig. 9 Flat plate with thickness variation linear buckling load improvement, with constant mass 


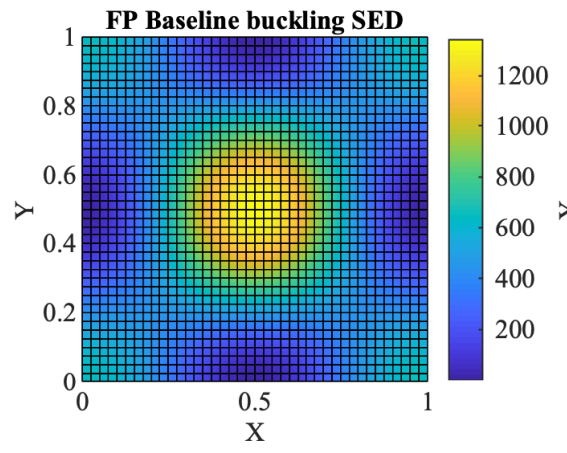

(a) Baseline result

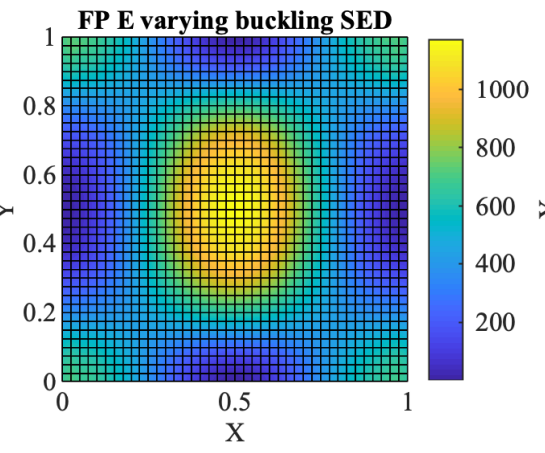

(b) Result with varied $E$

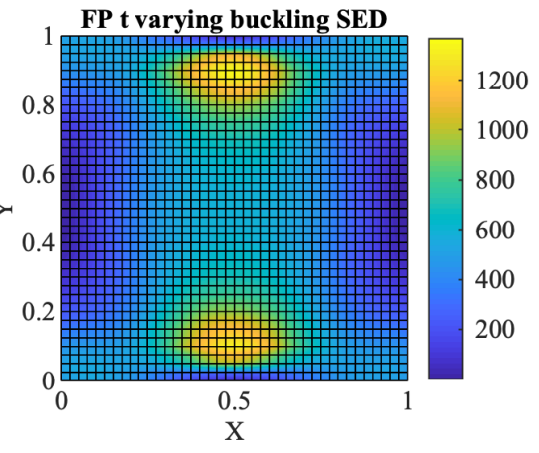

(c) Result with varied thickness

Fig. 10 Strain energy density of the first buckling mode (compression in $y$ ), outer surface, $\mathbf{J m}^{-1}$

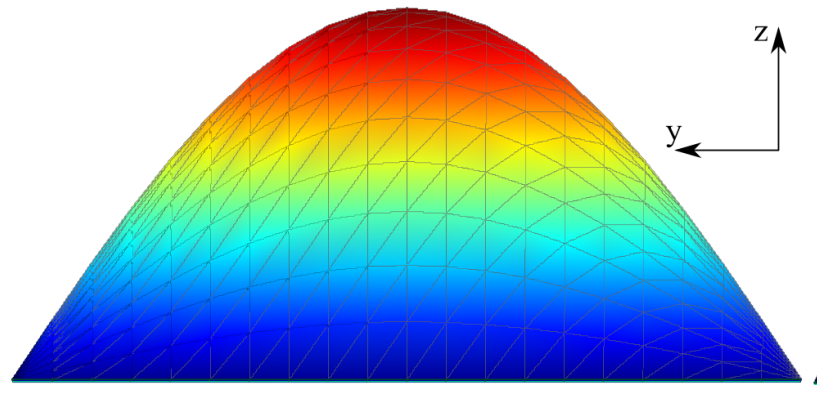

(a) Baseline

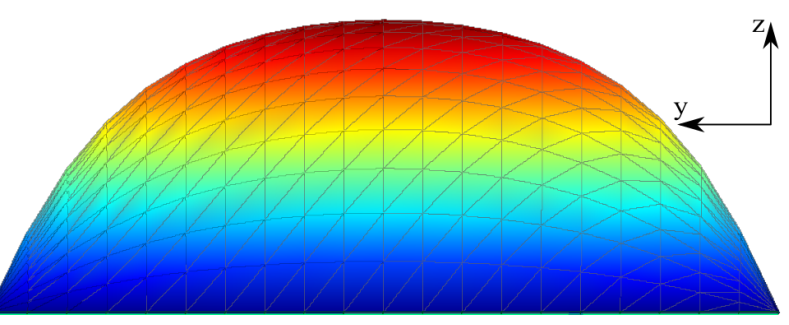

(b) With thickness redistribution

Fig. 11 Linear buckling mode of baseline and thickness varied structure, relative displacement (projection facing unloaded edge) 


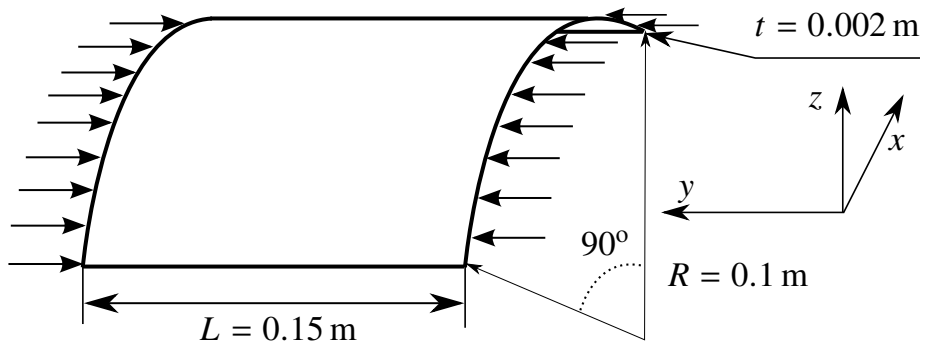

(a) Force and geometry

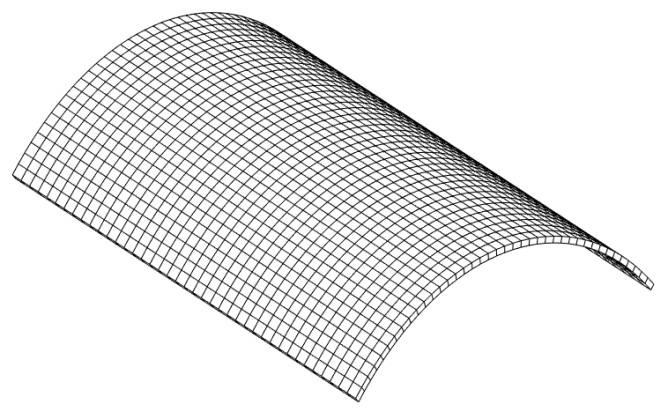

(b) Curved panel mesh

Fig. 12 Curved panel structure

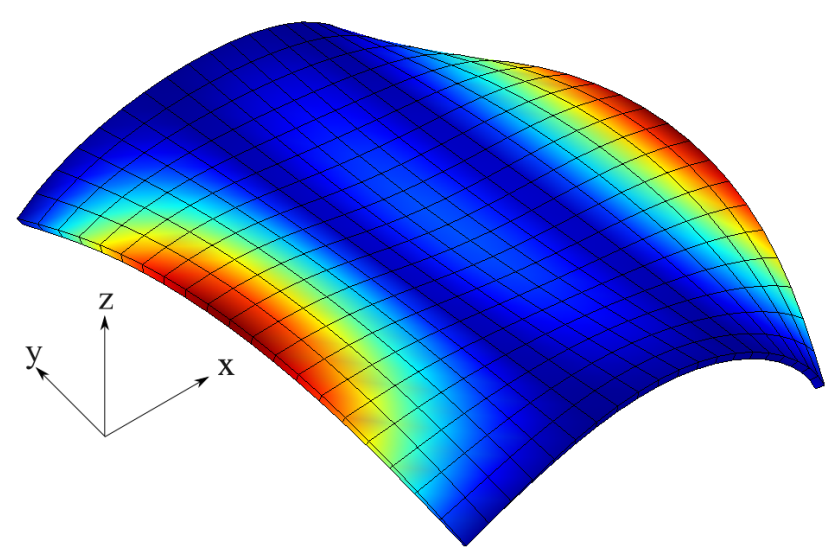

Fig. 13 First linear eigenmode of the curved panel, compression in $y$

\section{B. Curved panel}

As a second example the curved panel shown in fig. $12 \mathrm{a}$ is analyzed. It is a $90^{\circ}$ arc of a cylindrical shell with a radius of $0.1 \mathrm{~m}$, thickness of $2 \mathrm{~mm}$ and a length of $0.15 \mathrm{~m}$. A uniformly distributed pressure load $1 \mathrm{~N}$ in magnitude is applied on the curved edges of the panel, while restricting displacements in the plane of the loaded edges. These boundary conditions differ from an earlier version of this paper [53], in which the same boundary conditions were used for the prebuckling state but had the loaded edges clamped during the eigenvalue analysis. To restrict the rigid body mode in the load direction two points on the ends of the curve at $y=0.075 \mathrm{~m}$ are fixed in the load direction. A discretization of $40 \mathrm{SL}$ elements in radial direction and 40 SL elements in the axial direction was used. Material properties of the previous example ( $E=181 \mathrm{GPa}, v=0.3$ ) are also used in this example. Using the CUF implementation the first buckling load of $102504 \mathrm{~N}$ was calculated, and verified using DIANA [50] with a 930 Q20SH element shell model (107280 N). Figure 13 shows the first linear buckling mode, calculated using the CUF implementation.

\section{Young's modulus variation}

A correlation pattern was generated using a total of 10000 random samples using a correlation length of $0.01 \mathrm{~m}$. During postprocessing the correlation of the structure's Young's modulus with the first buckling mode was computed, as 


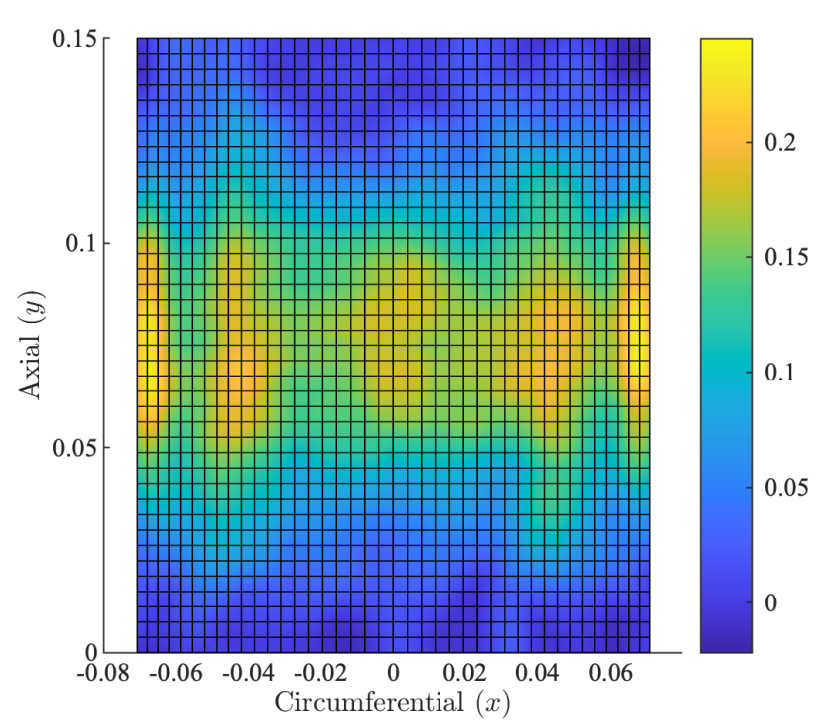

(a) Correlation of $E$ with first linear buckling load

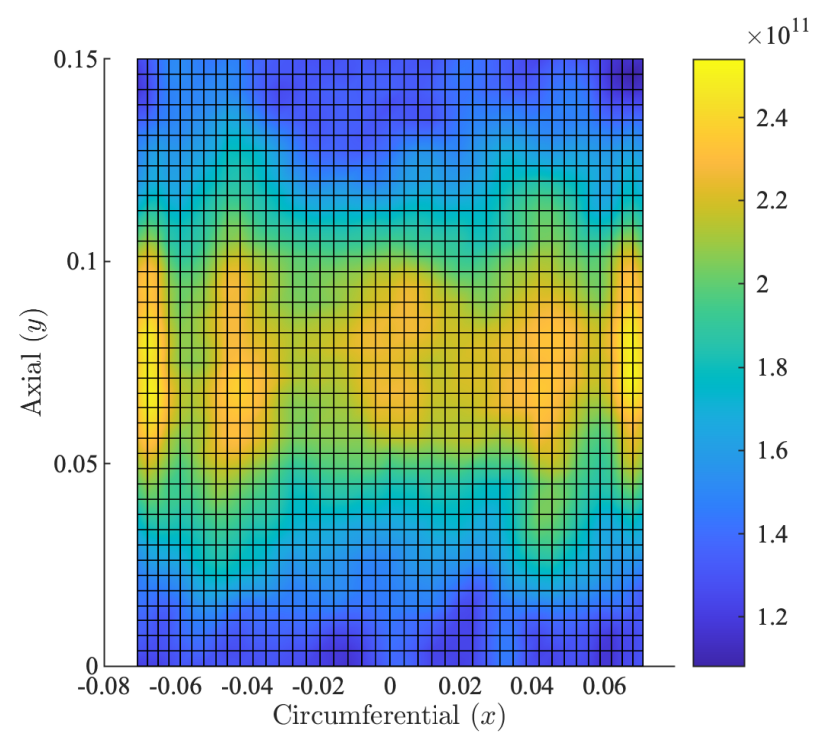

(b) Young's modulus $(m=0.78), \mathbf{P a}$

Fig. 14 Calculated correlation pattern and applied stiffness distribution

shown in fig. 14a An examination of the pattern shows that the panel benefits the most from reinforcements in the center of the straight edges, in the area of the maximum buckling amplitude. Stiffened regions are focused into five zones around the center. As with the flat panel, the increase was studied under a range of minima and maxima. The trends of these analyses are shown in fig. 15. Utilizing the full range of 108-254 GPa the material distribution of fig. 14b shows the largest increase in linear buckling load. Using this redistributed stiffness results in a $7.4 \%$ increase in linear buckling load to $113820 \mathrm{~N}$. Applying this stiffness redistribution also causes the prebuckling deformation to change, as is shown in fig. $16 \mathrm{~b}$

\section{Thickness variation}

Thickness variations are analyzed by running 10000 linear buckling analyses in which the mesh is perturbed proportional to the thickness by a 5\% standard deviation. The load vector is updated at every sample, updating makes sure that magnitude on an edge remains unity, and the load is distributed evenly on the surface. Variations are applied to the structure using a correlation length of $0.01 \mathrm{~m}$. Figure $18 \mathrm{a}$ shows the pattern of these correlations. A study was done to analyze the improvement in linear buckling load, the resulting trends are shown in fig. 20. In these analyses the thickness range was changed together with an appropriate scaling parameter $m$, while retaining the original mesh volume (mass). The greatest increase was found using a range of $-50 \%$ to $15 \%$ of the original thickness, using a scaling factor $m=1.56$. As was the case with the flat plate example the optimal range is a tradeoff between localized bending stiffness increase and a reduction elsewhere in the structure (due to the constant mass constraint imposed). Applying these ranges improved the linear buckling load by 29\%, to 136702 N. Figure $18 \mathrm{~b}$ shows the corresponding thickness distribution. 


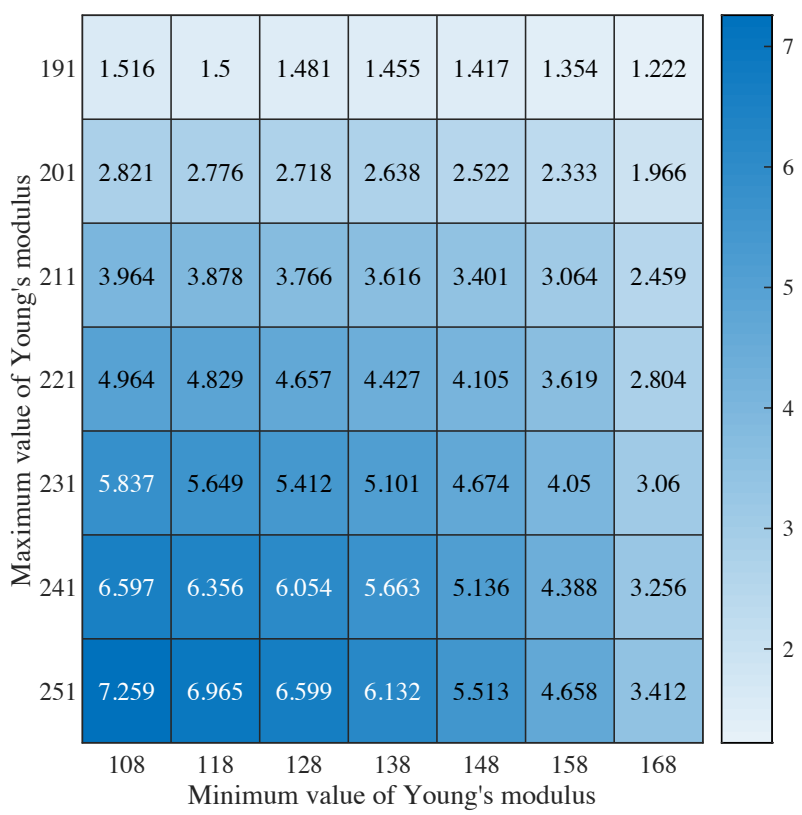

(a) Percentage increase in linear buckling load, \%

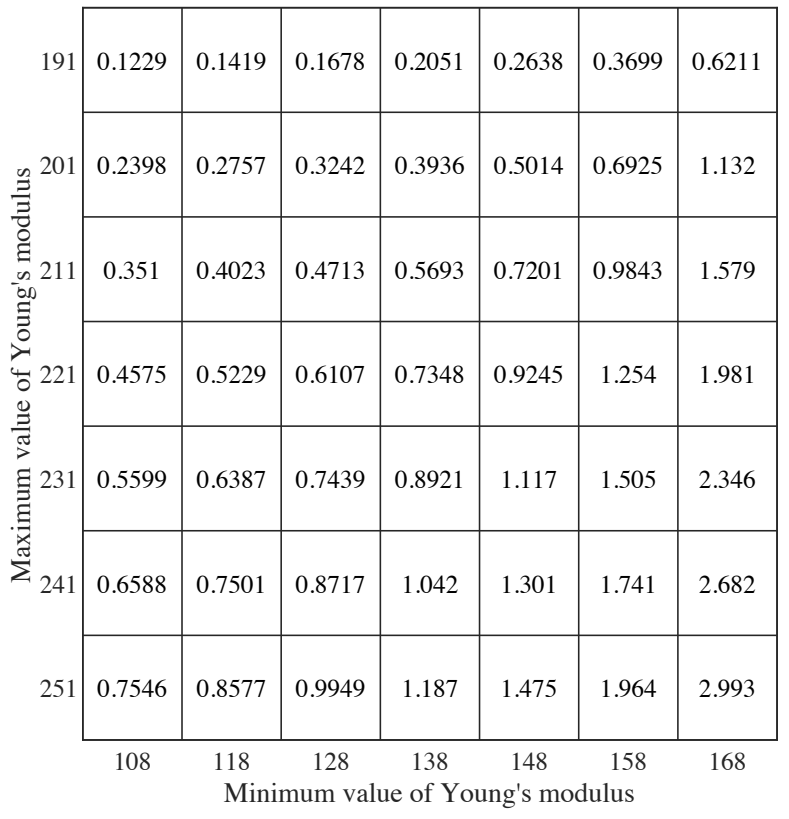

(b) Parameter $m$ (eq. 24) for constant Young's modulus

Fig. 15 Improvement over a range of Young's modulus ranges of the curved panel, with average value equaling the baseline Young's modulus

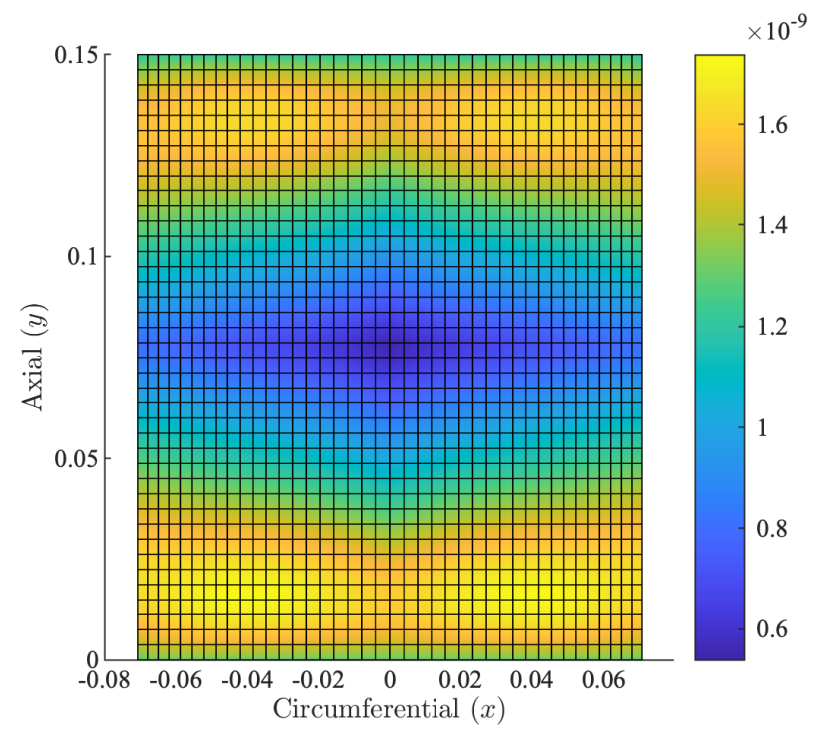

(a) Baseline

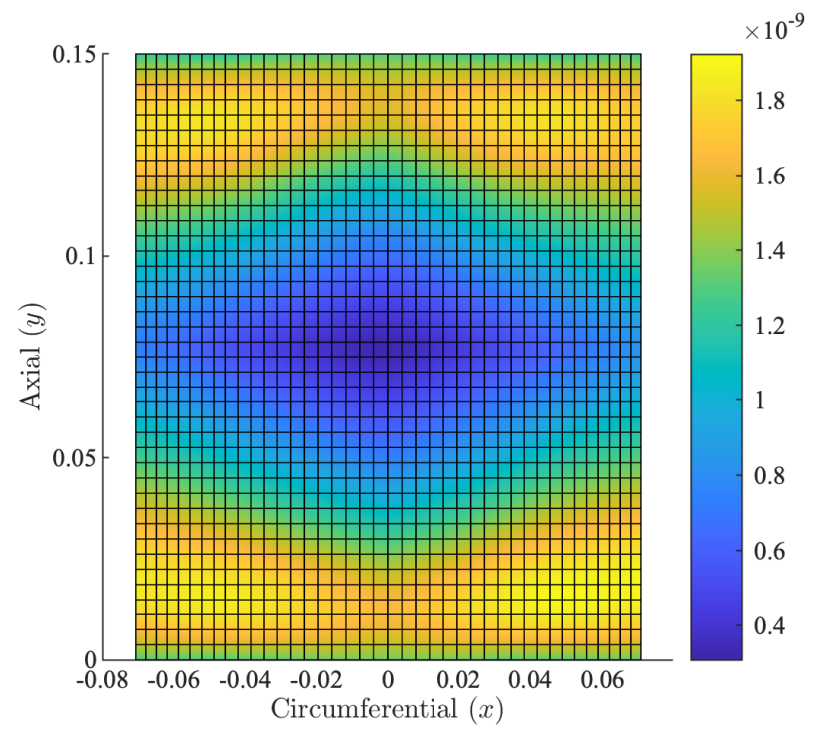

(b) With Young's modulus redistribution

Fig. 16 Prebuckling displacement of curved panel, $m$ 


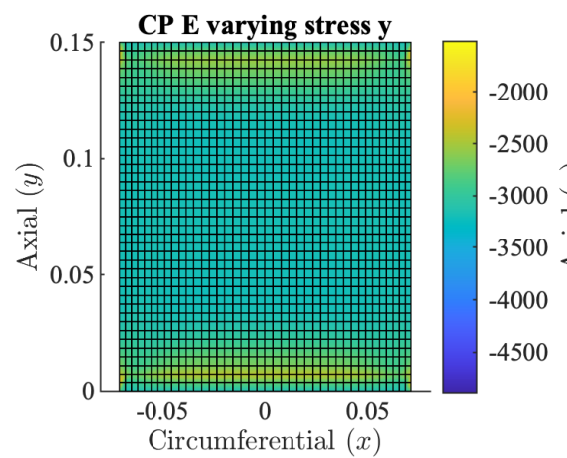

(a) Baseline result

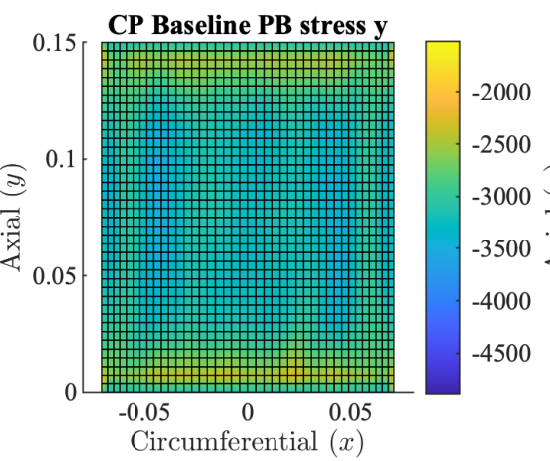

(b) Result with varied $E$

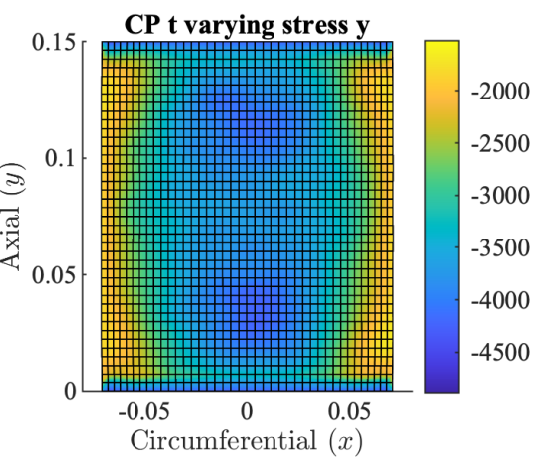

(c) Result with varied thickness

Fig. 17 Prebuckling stress in $y$ direction for three different curved panel analyses with compression in $y$, Pa

\section{Interpretation}

As was done for the flat plate the interpretation focused on the prebuckling stress and the strain energy density of the buckling mode. Changes in the prebuckling stress in the load (y) direction reduce in areas where buckles form, particularly for the thickness varying structure, as shown in fig. $17 \mathrm{c}$. At the same time the buckling strain energy density $\left(U=\frac{1}{2} \varepsilon_{i j} \sigma_{i j}[52\right.$, p. 122] $)$ of the structures has also redistributed. The original structure had a concentration of strain energy along the unsupported edges, which decreased in the Young's modulus tailored structure, and even more so in the thickness tailored structure. Changes in the prebuckling stress pattern are more prominent than changes in strain energy density, which differs from the previous example. Increases in buckling strain energy density at the corners of the thickness tailored structure is due to the local decrease in thickness, which cause a redistribution of the applied force. This influences the load path of forces applied to the structure, allowing these to be focused in areas less sensitive to high stresses.

\section{Effect of correlation length}

Patterns are generated using random variations of the Young's modulus or thickness throughout the structure. Variations are correlated to each other, meaning that points close to each other are related, more so than points far apart from each other. Patterns of correlations shown in this section are similarly related. A comparison of the curved panel thickness example is shown in fig. 21, where the thickness correlation of the curved panel is shown for a correlation length of 0.05 and $0.01 \mathrm{~m}$. Both analyses are run 10000 times, but it is clear that the $0.05 \mathrm{~m}$ pattern appears to be more converged. It is also clear that the pattern with a correlation length of $0.01 \mathrm{~m}$ is different, preferring a fairly thin strip along the edges instead of the larger areas along the off center areas of the panel.

Similarly an analysis was run in which the flat panel analysis of section IV.A.1 was run with a correlation length of 1 m. A round pattern was generated instead of an oval pattern. The correlation length in this context can be interpreted as a characteristic pattern minimum feature length. A shorter length, while giving more resolution to the pattern also 


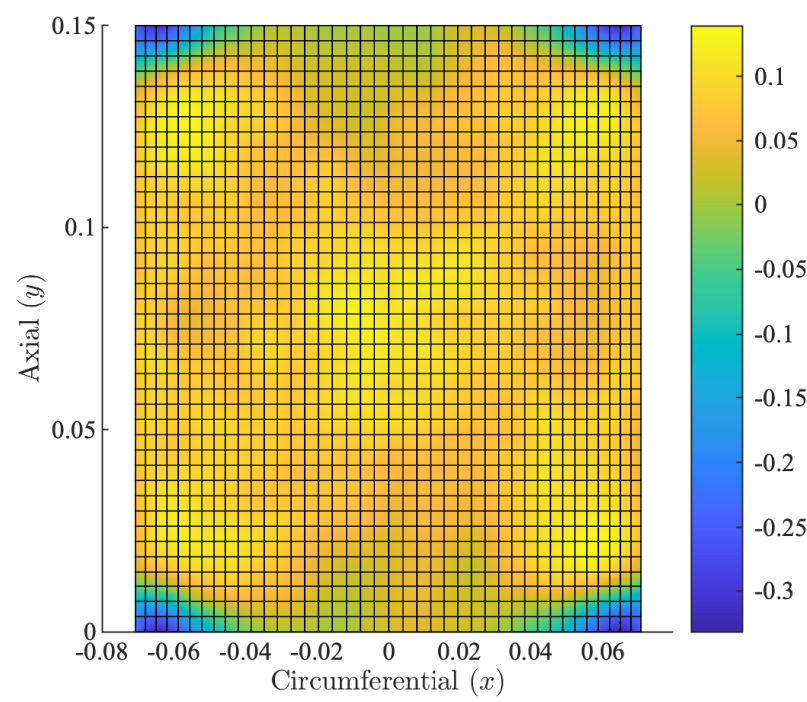

(a) Correlation of thickness with first linear buckling load

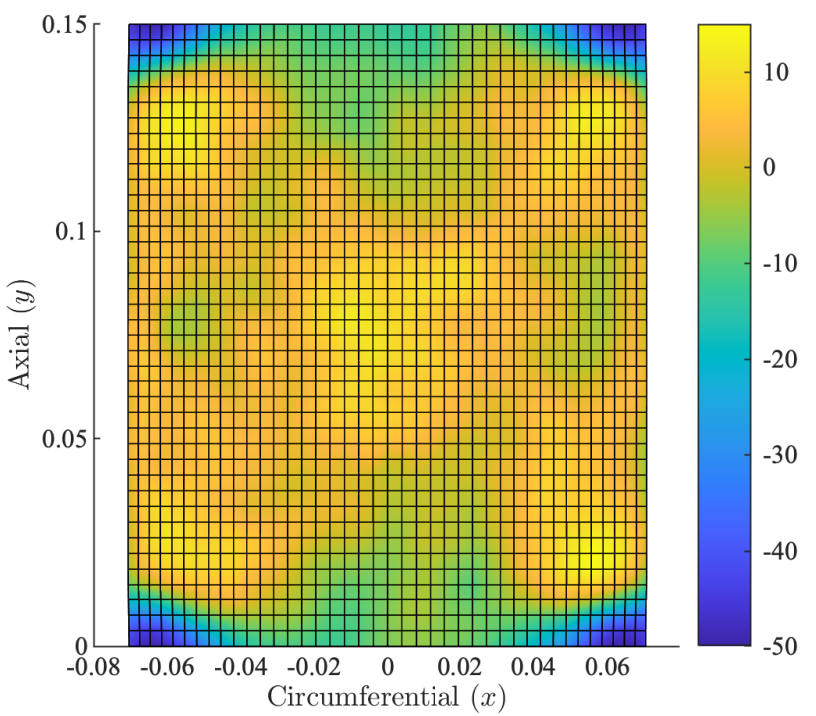

(b) Thickness change $(m=1.56), \%$ of original thickness

Fig. 18 Calculated correlation pattern and applied thickness distribution of curved panel

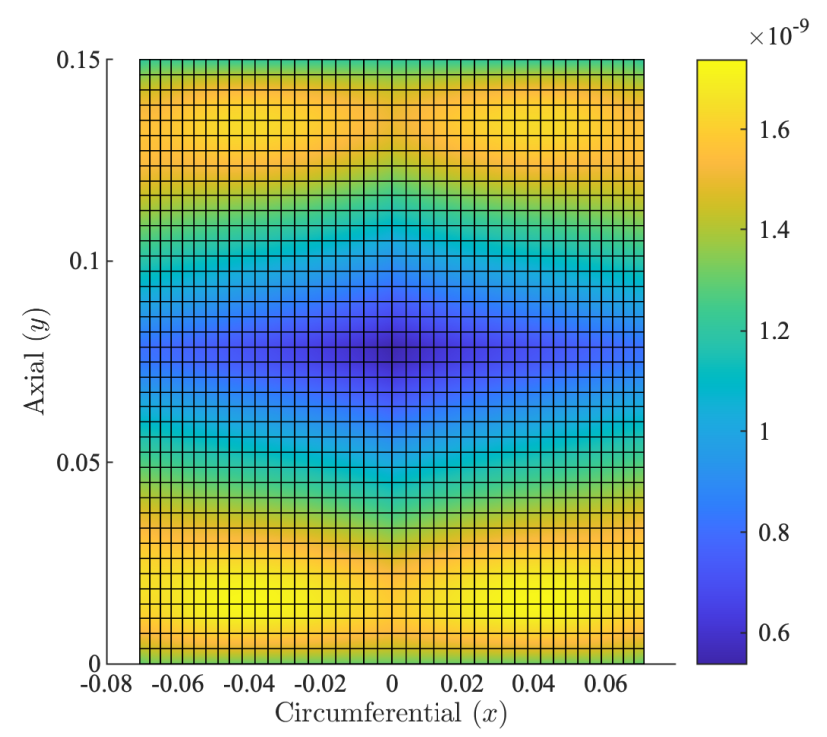

(a) Baseline

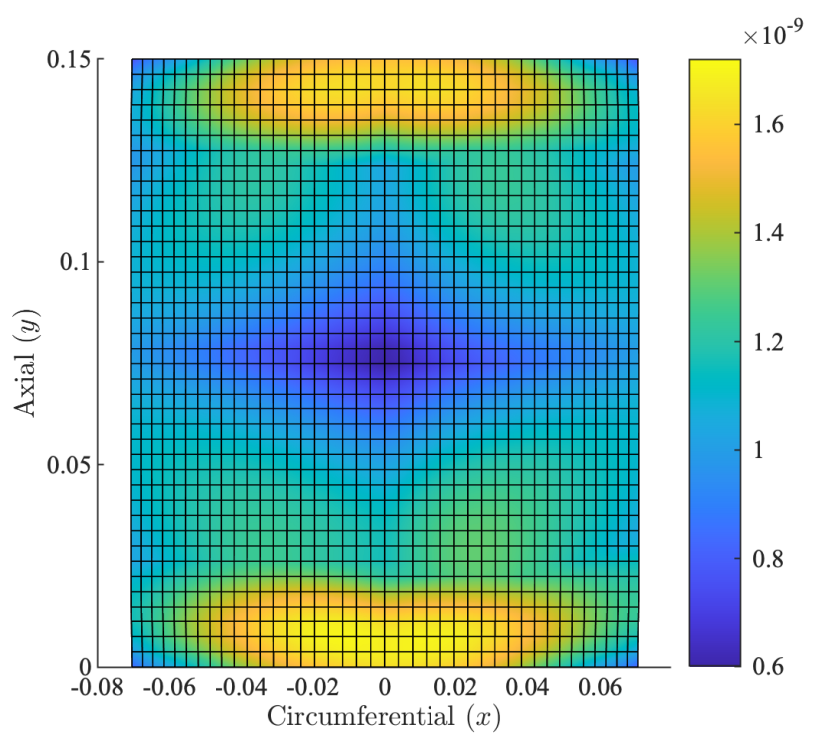

(b) With thickness distribution

Fig. 19 Prebuckling displacement of curved panel change with thickness variation, $m$ 


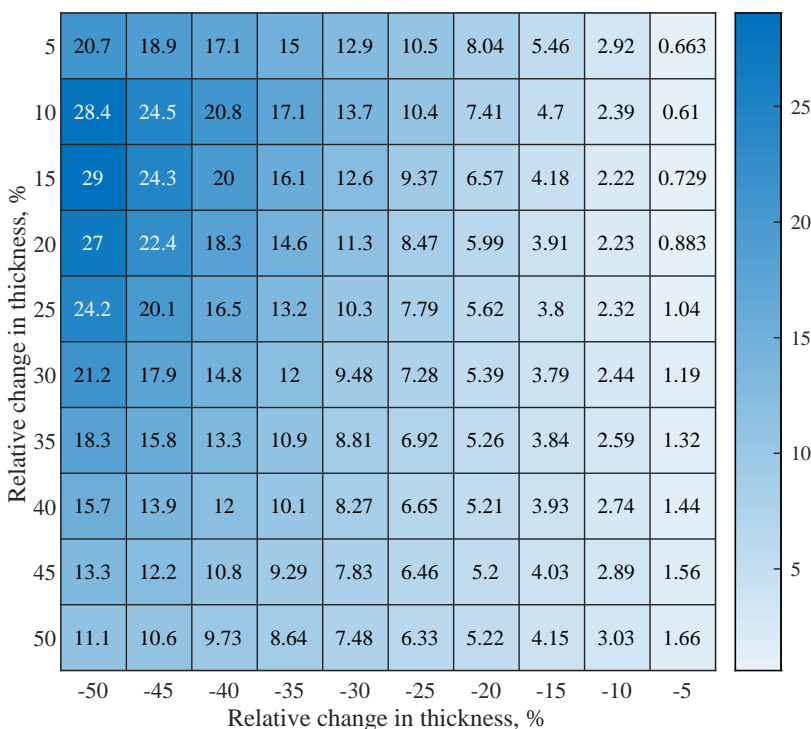

(a) Percentage increase in linear buckling load

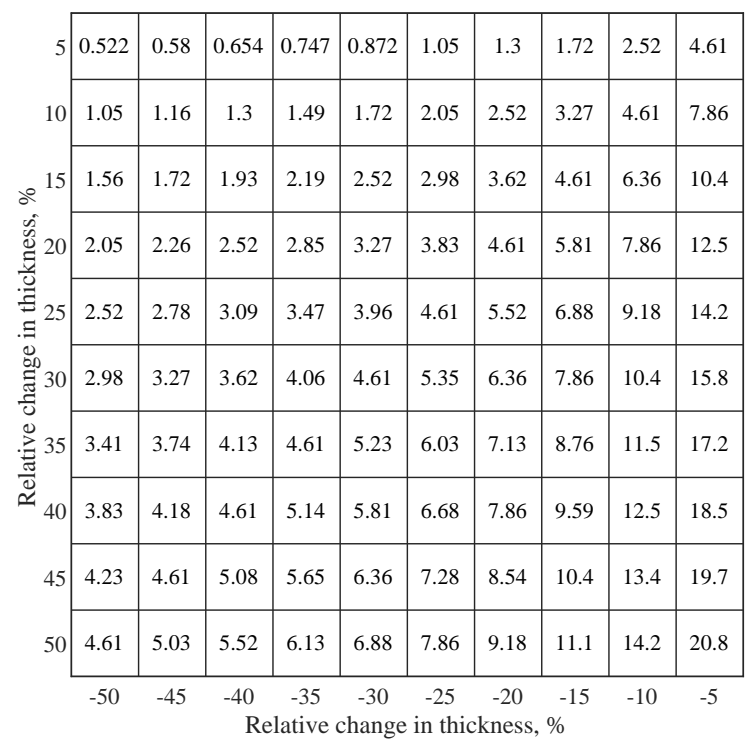

(b) Scaling parameter $m$ (eq. (24) for constant mass

Fig. 20 Improvement of buckling load of curved panel over a range of thickness ranges, with constant mass

causes the pattern to take longer to converge.

To analyze how the two patterns compare, analyses were done in which the thickness variation was compared. The $0.01 \mathrm{~m}$ pattern of fig. $21 \mathrm{a}$ shows an increase of $29 \%$ using $m=1.56$ over a range of change from $-50 \%$ to $15 \%$ of the original thickness. A similar analysis was done for a correlation length of $0.05 \mathrm{~m}$. The best improvement was found in the range $-50 \%$ to $15 \%$, which showed an increase of the linear buckling load of $9.4 \%$, using a scaling factor of $m=0.44$. Comparing this to the result generated with a smaller correlation length gives only a third of the increase. A shorter correlation length has a better potential enhancement, at a higher computational expense.

\section{Discussion, conclusions and future work}

Through stochastic analyses of randomized local variations of, for example, thickness it is possible to glean information on the average local sensitivity of the structure to a variation of that parameter. Using this information it is possible to tailor structures to enhance their mechanical response. Numerical examples have demonstrated such effects through examples which improve the linear buckling load of two structures through stiffness redistribution, either by varying the Young's modulus or thickness locally. The new stiffness distributions cause the bending stiffness to increase in areas where buckling deformation is most significant. Another effect is that the prebuckling stress tends to reduce in those areas, the combination of stiffness and corresponding stress redistributions is that the strain energy density of sensitive areas reduces. Analyses over multiple correlation lengths were accomplished. An overall trend shows correlation patterns with a shorter length generate patterns which perform better. This result is due to the pattern having a higher resolution (i.e. is less smooth), allowing enhancements to be more localized. 


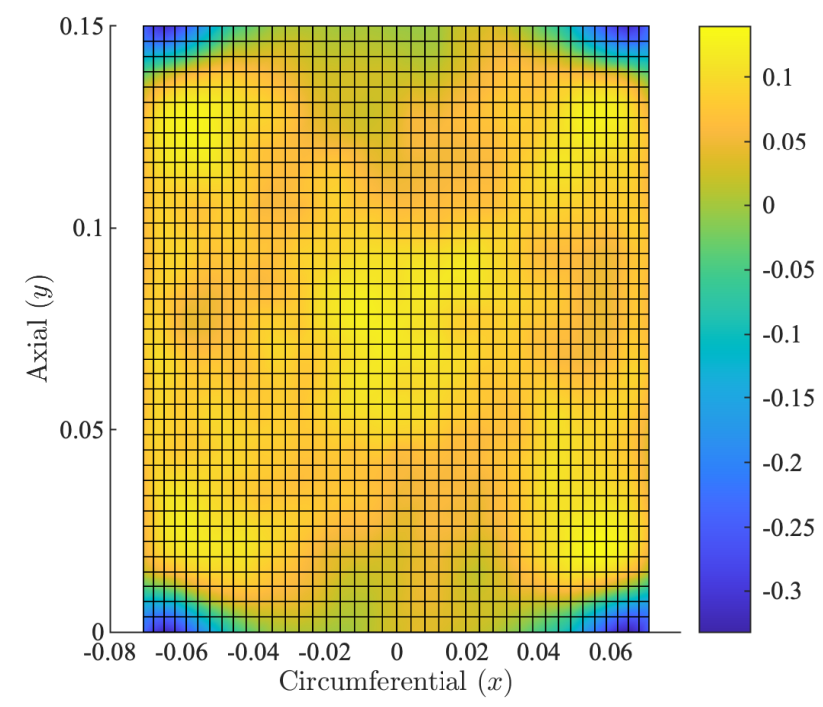

(a) Correlation of thickness with linear buckling load $L_{c}=$ $0.01 \mathrm{~m}$

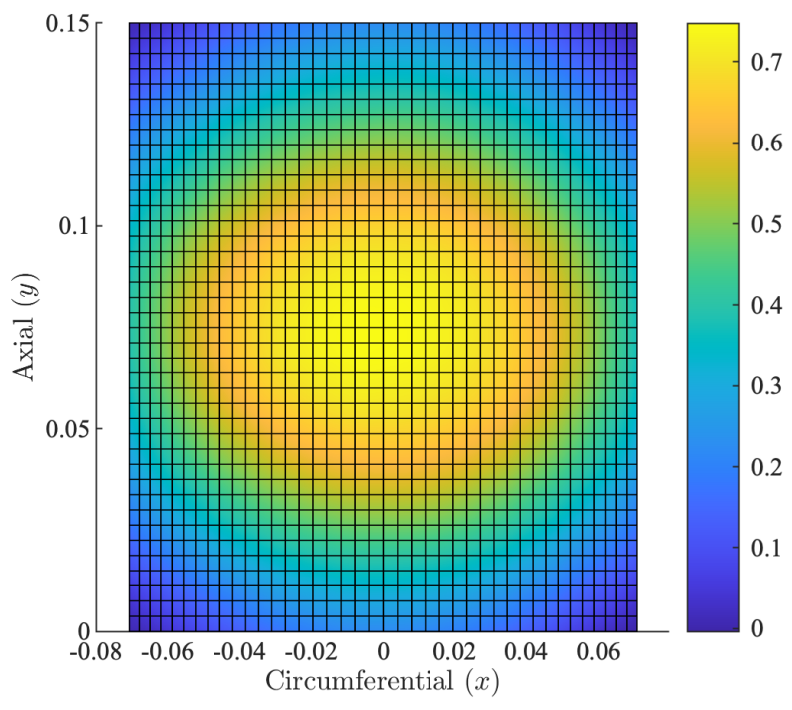

(b) Correlation of thickness with linear buckling load $L_{c}=$ $0.05 \mathrm{~m}$

Fig. 21 Comparison to correlation patterns of thickness variations of curved panel with different correlation lengths

Correlation patterns are different depending on which parameter (e.g. Young's modulus or thickness) is varied, in the examples shown this is due to two effects, the first being the effect variations have on the stiffness. Both parameters increase in-plane stiffness linearly with their variation. Bending stiffness on the other hand is proportional to $E t^{3}$, thickness variations therefore affect the bending stiffness of the structure much more than varying the Young's modulus. Variations of the thickness cause another effect, force is applied to the edge of the structure as a distributed load. When the thickness decreases the load is redistributed away from the area and into other areas (as the area decreases locally), this can help redirect load to parts of the structure that are less sensitive. The corners of the curved panel depicts this, these areas are thinner so as to decrease the relative amount of load applied in those areas close to the edge. In this way the thickness distribution also tailors the load path while tailoring the stiffness.

Future extensions can broaden the use of the method. Local stress amplitude reduction during cyclic loading could also be decreased, which could lead to an improved fatigue life. The results so far are limited to linear results, it is also possible run these analyses using non-linear analyses at a specific load level or buckling load.

Potential use of this approach on additive manufactured materials and structures is also of interest. Extra design freedoms facilitated by these production processes makes it possible to create complex geometry, which would not be economical with traditional manufacturing processes. When the manufacturing process is well understood and the variations can be quantified in a representative fashion the effects on the structural performance of the tailored structure can be directly compared, potentially enabling the structure simultaneously to be more highly tailored and have excellent performance through the same design process. 
Adding multiple design iterative stages can also lead to further improvement of linear, and potentially non-linear problems. Results shown in figs. 9a and 20a indicate that maximizing the parameter range does not automatically lead to the best improvement. To find an optimal distributions successive iterations will likely be needed. By applying the random fields to out-of-plane displacements it may also be possible to add tailored variations to affect the behavior. Fields can be generated in 2D, as done here, but random fields can also be generated for 3D structures. Optimization using these methods does not necessarily require a converged correlation pattern, the patterns could be perturbed and modified in successive iterations each time an improvement is found. It is noted that convergence would likely improve by postprocessing on the patterns, utilizing symmetry for instance.

\section{Acknowledgments}

This project has received funding from the European Union's Horizon 2020 research and innovation program under the Marie Skłodowska-Curie grant agreement No. 642121.

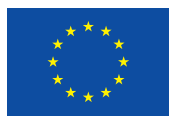

This work was carried out using the computational facilities of the Advanced Computing Research Centre, University of Bristol - http://www.bris.ac.uk/acrc/ as well as computational facilities within the Institute of Structural Analysis at Leibniz University Hannover.

Paul M. Weaver would like to thank the Science Foundation Ireland for support under its Research Professor scheme (Varicomp:15/RP/2773) and the Royal Society for its Wolfson Merit Award.

\section{References}

[1] Stefanou, G., "The stochastic finite element method: Past, present and future," Computer Methods in Applied Mechanics and Engineering, Vol. 198, No. 9-12, 2009, pp. 1031-1051. doi:10.1016/j.cma.2008.11.007, URL http://dx.doi .org/10. $1016 / \mathrm{j} . \mathrm{cma} .2008 .11 .007$

[2] Ghanem, R. G., and Spanos, P. D., “Spectral Stochastic Finite-Element Formulation For Reliability Analysis,” Journal of Engineering Mechanics, Vol. 117, No. 10, 1991, pp. 2351-2372. doi:10.1061/(ASCE)0733-9399(1991)117:10(2351), URL http://doi.org/10.1061/(ASCE)0733-9399(1991)117:10(2351)

[3] Der Kiureghian, A., and Ke, J.-B., "The stochastic finite element method in structural reliability,” Probabilistic Engineering Mechanics, Vol. 3, No. 2, 1988, pp. 83-91. doi:10.1016/0266-8920(88)90019-7, URL http://doi .org/10.1016/0266$8920(88) 90019-7$

[4] Spanos, P. D., and Zeldin, B. A., "Monte Carlo Treatment of Random Fields: A Broad Perspective," Applied Mechanics Reviews, Vol. 51, No. 3, 1998, p. 219. doi:10.1115/1.3098999, URLhttp://doi.org/10.1115/1.3098999 
[5] Papadopoulos, V., Stefanou, G., and Papadrakakis, M., "Buckling analysis of imperfect shells with stochastic non-Gaussian material and thickness properties," International Journal of Solids and Structures, Vol. 46, No. 14-15, 2009, pp. $2800-2808$. doi:10.1016/j.ijsolstr.2009.03.006, URL http://dx.doi.org/10.1016/j.ijsolstr.2009.03.006

[6] Vořechovský, M., and Novák, D., "Simulation of random fields for stochastic finite element analysis," Icossar, 2005, pp. 2545-2552. URL https://pdfs.semanticscholar.org/0570/dac368ae7d1d6d980ecc78bf25d8b75585b6.pdf

[7] Vryzidis, I., Stefanou, G., and Papadopoulos, V., "Stochastic stability analysis of steel tubes with random initial imperfections," Finite Elements in Analysis and Design, Vol. 77, 2013, pp. 31-39. doi:10.1016/j.finel.2013.09.002, URL http://dx.doi. org/10.1016/j.finel.2013.09.002

[8] Tootkaboni, M., Graham-Brady, L., and Schafer, B. W., "Geometrically non-linear behavior of structural systems with random material property: An asymptotic spectral stochastic approach," Computer Methods in Applied Mechanics and Engineering, Vol. 198, No. 37-40, 2009, pp. 3173-3185. doi:10.1016/j.cma.2009.05.014, URL http://dx.doi.org/10.1016/j.cma. 2009.05 .014

[9] Broek, S. v. d., Jansen, E., Minera, S., Weaver, P. M., and Rolfes, R., "Effect of spatially varying material properties on the post-buckling behaviour of composite panels utilising geodesic stochastic fields," Proceedings of the 6th Aircraft Structural Design conference, Bristol, 2018.

[10] Sasikumar, P., Suresh, R., Vijayaghosh, P. K., and Gupta, S., "Experimental characterisation of random field models for CFRP composite panels," Composite Structures, Vol. 120, 2015, pp. 451-471. doi:10.1016/j.compstruct.2014.10.023, URL http://dx.doi.org/10.1016/j.compstruct.2014.10.023

[11] Sriramula, S., and Chryssanthopoulos, M. K., "An experimental characterisation of spatial variability in GFRP composite panels," Structural Safety, Vol. 42, 2013, pp. 1-11. doi:10.1016/j.strusafe.2013.01.002, URL http://dx.doi . org/10.1016/ j.strusafe.2013.01.002

[12] Sriramula, S., and Chryssanthopoulos, M. K., "Quantification of uncertainty modelling in stochastic analysis of FRP composites," Composites Part A: Applied Science and Manufacturing, Vol. 40, No. 11, 2009, pp. 1673-1684. doi:10.1016/j.compositesa. 2009.08.020, URL http://dx.doi.org/10.1016/j.compositesa.2009.08.020.

[13] Lekou, D. J., and Philippidis, T. P., "Mechanical property variability in FRP laminates and its effect on failure prediction," Composites Part B: Engineering, Vol. 39, No. 7-8, 2008, pp. 1247-1256. doi:10.1016/j.compositesb.2008.01.004, URL http://doi.org/10.1016/j.compositesb.2008.01.004

[14] Saenz-Castillo, D., Martín, M., Calvo, S., Rodriguez-Lence, F., and Güemes, A., "Effect of processing parameters and void content on mechanical properties and NDI of thermoplastic composites," Composites Part A: Applied Science and Manufacturing, Vol. 121, No. January, 2019, pp. 308-320. doi:10.1016/J.COMPOSITESA.2019.03.035, URL http: //doi.org/10.1016/J.COMPOSITESA.2019.03.035 
[15] Manta, A., Gresil, M., and Soutis, C., "Infrared thermography for void mapping of a graphene/epoxy composite and its full-field thermal simulation," Fatigue and Fracture of Engineering Materials and Structures, , No. October 2018, 2019 , pp. 1-13. doi:10.1111/ffe.12980, URL http://doi.org/10.1111/ffe.12980

[16] Gurdal, Z., and Olmedo, R., "In-plane response of laminates with spatially varying fiber orientations - Variable stiffness concept," AIAA Journal, Vol. 31, No. 4, 1993, pp. 751-758. doi:10.2514/3.11613, URL http://doi.org/10.2514/3.11613.

[17] Blom, A. W., Stickler, P. B., and Gürdal, Z., "Optimization of a composite cylinder under bending by tailoring stiffness properties in circumferential direction," Composites Part B: Engineering, Vol. 41, No. 2, 2010, pp. 157-165. doi:10.1016/j. compositesb.2009.10.004, URL http://dx.doi.org/10.1016/j.compositesb.2009.10.004

[18] Burgueño, R., Hu, N., Heeringa, A., and Lajnef, N., "Tailoring the elastic postbuckling response of thin-walled cylindrical composite shells under axial compression,” Thin-Walled Structures, Vol. 84, 2014, pp. 14-25. doi:10.1016/j.tws.2014.05.009, URL http://dx.doi.org/10.1016/j.tws.2014.05.009.

[19] Chi, S. H., and Chung, Y. L., "Mechanical behavior of functionally graded material plates under transverse load-Part I: Analysis," International Journal of Solids and Structures, Vol. 43, No. 13, 2006, pp. 3657-3674. doi:10.1016/j.ijsolstr.2005.04.011, URL http://doi.org/10.1016/j.ijsolstr.2005.04.011

[20] $\mathrm{Hu}, \mathrm{N}$. , and Burgueño, R., "Elastic postbuckling response of axially-loaded cylindrical shells with seeded geometric imperfection design," Thin-Walled Structures, Vol. 96, No. September, 2015, pp. 256-268. doi:10.1016/j.tws.2015.08.014, URL http://dx.doi.org/10.1016/j.tws.2015.08.014.

[21] Cox, B. S., Groh, R. M., Avitabile, D., and Pirrera, A., "Modal nudging in nonlinear elasticity: Tailoring the elastic postbuckling behaviour of engineering structures," Journal of the Mechanics and Physics of Solids, Vol. 116, 2018, pp. 135-149. doi:10.1016/j.jmps.2018.03.025, URL https://doi .org/10.1016/j .jmps.2018.03.025

[22] Hu, N., and Burgueño, R., "Harnessing Seeded Geometric Imperfection to Design Cylindrical Shells With Tunable Elastic Postbuckling Behavior,” Journal of Applied Mechanics, Vol. 84, No. 1, 2017, p. 011003. doi:10.1115/1.4034827, URL http://doi.org/10.1115/1.4034827

[23] Hu, N., and Burgueño, R., "Cylindrical Shells with Tunable Postbuckling Features Through Non-Uniform Patterned Thickening Patches," International Journal of Structural Stability and Dynamics, Vol. 18, No. 02, 2018, p. 1850026. doi:10.1142/ S0219455418500268, URL http://doi.org/10.1142/S0219455418500268

[24] Li, Y. W., Elishakoff, I., and Starnes, J. H., "Axial buckling of composite cylindrical shells with periodic thickness variation," Computers and Structures, Vol. 56, No. 1, 1995, pp. 65-74. doi:10.1016/0045-7949(94)00527-A, URL http: //doi.org/10.1016/0045-7949(94)00527-A

[25] Chen, Z., Yang, L., Cao, G., and Guo, W., "Buckling of the axially compressed cylindrical shells with arbitrary axisymmetric thickness variation," Thin-Walled Structures, Vol. 60, 2012, pp. 38-45. doi:10.1016/j.tws.2012.07.015, URL http://dx.doi. org/10.1016/j.tws.2012.07.015. 
[26] Guest, J. K., "Optimizing the layout of discrete objects in structures and materials: A projection-based topology optimization approach," Computer Methods in Applied Mechanics and Engineering, Vol. 283, 2015, pp. 330-351. doi:10.1016/j.cma.2014. 09.006, URL http://dx.doi.org/10.1016/j.cma.2014.09.006

[27] Li, Y. W., Elishakoff, I., Starnes, J. H., and Bushnell, D., "Effect of the thickness variation and initial imperfection on buckling of composite cylindrical shells: Asymptotic analysis and numerical results by BOSOR4 and PANDA2," International Journal of Solids and Structures, Vol. 34, No. 28, 1997, pp. 3755-3767. doi:10.1016/S0020-7683(96)00230-2, URL http://doi.org/10.1016/S0020-7683(96)00230-2

[28] Lagaros, N. D., and Papadopoulos, V., "Optimum design of shell structures with random geometric, material and thickness imperfections," International Journal of Solids and Structures, Vol. 43, No. 22-23, 2006, pp. 6948-6964. doi:10.1016/j.ijsolstr. 2006.02.019, URL http://doi.org/10.1016/j.ijsolstr.2006.02.019

[29] Sliseris, J., and Rocens, K., “Optimal design of composite plates with discrete variable stiffness,” Composite Structures, Vol. 98, 2013, pp. 15-23. doi:10.1016/j.compstruct.2012.11.015, URL http://dx.doi.org/10.1016/j.compstruct.2012.11. Q15.

[30] Lee, K. H., and Park, G. J., "Robust optimization considering tolerances of design variables," Computers and Structures, Vol. 79, No. 1, 2001, pp. 77-86. doi:10.1016/S0045-7949(00)00117-6, URLhttp://doi .org/10 .1016/S0045-7949(00)00117-6

[31] Chen, X., Hasselman, T., Neill, D., Chen, X., Hasselman, T., and Neill, D., "Reliability based structural design optimization for practical applications,”, No. 1, 2013. doi:10.2514/6.1997-1403, URL http://doi.org/10.2514/6.1997-1403

[32] Guo, X., Zhang, W., and Zhang, L., "Robust structural topology optimization considering boundary uncertainties," Computer Methods in Applied Mechanics and Engineering, Vol. 253, 2013, pp. 356-368. doi:10.1016/j.cma.2012.09.005, URL http://dx.doi.org/10.1016/j.cma.2012.09.005

[33] Minera, S., “Analysis and Design of Buckling Resistant Thin-Walled Structures via Computationally Efficient 3D Stress Analysis,” Ph.D. thesis, University of Bristol, 2019.

[34] Carrera, E., Cinefra, M., Petrolo, M., and Zappino, E., Finite element analysis of structures through unified formulation, Wiley Online Library, 2014. doi:10.1002/9781118536643, URL http://doi .org/10 .1002/9781118536643

[35] Minera, S., Patni, M., Carrera, E., Petrolo, M., Weaver, P. M., and Pirrera, A., "Three-dimensional stress analysis for beam-like structures using Serendipity Lagrange shape functions," International Journal of Solids and Structures, Vol. 141-142, 2018, pp. 279-296. doi:10.1016/j.ijsolstr.2018.02.030, URL http://doi.org/10.1016/j.ijsolstr.2018.02.030

[36] Patni, M., “An Efficient Numerical Framework for Capturing Localised 3D Stress Fields in Laminated Composites,” Ph.D. thesis, University of Bristol, 2019.

[37] Carrera, E., Giunta, G., and Petrolo, M., Beam Structures: Classical and Advanced Theories, John Wiley \& Sons, Ltd, Chichester, UK, 2011. doi:10.1002/9781119978565, URL http://doi.org/10.1002/9781119978565 
[38] Pagani, A., and Carrera, E., "Unified formulation of geometrically nonlinear refined beam theories," Mechanics of Advanced Materials and Structures, Vol. 25, No. 1, 2018, pp. 15-31. doi:10.1080/15376494.2016.1232458, URL https://doi .org/ $10.1080 / 15376494.2016 .1232458$

[39] Patni, M., Minera, S., Bisagni, C., Weaver, P., and Pirrera, A., "Geometrically nonlinear finite element model for predicting failure in composite structures," Composite Structures, Vol. 225, No. May, 2019, p. 111068. doi:10.1016/j.compstruct.2019.111068, URL https://doi.org/10.1016/j.compstruct.2019.111068

[40] Cook, R. D., Malkus, D. S., Plesha, M. E., and Witt, R. J., Concepts and applications of finite element analysis, $4^{\text {th }}$ ed., Vol. 4 , Wiley New York, 2002. URL/https://www.wiley.com/en-us/Concepts+and+Applications+of+Finite+Element+ Analysis\%2C+4th+Edition-p-9780471356059.

[41] Pagani, A., de Miguel, A. G., and Carrera, E., "Cross-sectional mapping for refined beam elements with applications to shell-like structures," Computational Mechanics, Vol. 59, No. 6, 2017, pp. 1031-1048. doi:10.1007/s00466-017-1390-7, URL http://doi.org/10.1007/s00466-017-1390-7.

[42] Szabó, B., and Babuška, I., Introduction to finite element analysis: formulation, verification and validation, Vol. 35, John Wiley \& Sons, 2011. doi:10.1002/9781119993834, URL http://doi.org/10.1002/9781119993834.

[43] Geuzaine, C., and Remacle, J. F., "Gmsh: A 3-D finite element mesh generator with built-in pre- and post-processing facilities," International Journal for Numerical Methods in Engineering, Vol. 79, No. 11, 2009, pp. 1309-1331. doi:10.1002/nme.2579, URL http://doi.org/10.1002/nme.2579

[44] Davis, M. W., "Production of conditional simulations via the LU triangular decomposition of the covariance matrix," Mathematical Geology, Vol. 19, No. 2, 1987, pp. 91-98. doi:10.1007/BF00898189, URL http://doi.org/10.1007/ BF00898189

[45] Sudret, B., and Kiureghian, a. D., "Stochastic Finite Element Methods and Reliability: A State-of-the-Art Report," University of California Berkeley, , No. November, 2000, p. 189. URL/https://www1.ethz.ch/ibk/su/publications/Reports/SFEreport-Sudret.pdf

[46] Dekking, F. M., Kraaikamp, C., Lopuhaä, H. P., and Meester, L. E., A Modern Introduction to Probability and Statistics: Understanding why and how, Springer Science \& Business Media, 2005. doi:10.1007/1-84628-168-7, URL https: //doi.org/10.1007/1-84628-168-7

[47] Scarth, C., Adhikari, S., Cabral, P. H., Silva, G. H. C., Prado, A. P. d., Higino, P., Silva, G. H. C., and Prado, A. P. d., "Random field simulation over curved surfaces : Applications to computational structural mechanics," Computer Methods in Applied Mechanics and Engineering, Vol. 345, 2019, pp. 283-301. doi:10.1016/j.cma.2018.10.026, URL https://doi.org/10.1016/j.cma.2018.10.026

[48] Crane, K., Weischedel, C., and Wardetzky, M., "The Heat Method for Distance Computation,” Communications of the ACM, Vol. 60, No. 11, 2017, pp. 90-99. doi:0.1145/3131280, URL https://doi.org/10.1145/3131280 
[49] Li, C.-C., and Der Kiureghian, A., "Optimal discretization of random fields,” Journal of engineering mechanics, Vol. 119, No. 6, 1993, pp. 1136-1154. doi:10.1061/(ASCE)0733-9399(1993)119:6(1136), URL https://doi .org/10.1061/(ASCE)07339399(1993) 119:6(1136)

[50] Diana FEA, “DIANA Finite Element Analysis User's Manual Release 10.3,” Tech. rep., 2019. URL https://dianafea. com/manuals/d103/Diana.html

[51] Mahamood, R. M., and Akinlabi, E. T., "Laser metal deposition of functionally graded Ti6Al4V/TiC," Materials and Design, Vol. 84, 2015, pp. 402-410. doi:10.1016/j.matdes.2015.06.135, URL http://dx.doi .org/10.1016/j .matdes.2015.06. 135

[52] Sadd, M. H., Elasticity-Theory, Applications, and Numerics, $2^{\text {nd }}$ ed., Academic press, 2014. doi:10.1016/c2012-0-06981-5, URL https: //doi .org/10.1016/c2012-0-06981-5

[53] Broek, S. v. d., Minera, S., Pirrera, A., Weaver, P. M., Jansen, E. L., and Rolfes, R., "Enhanced Deterministic Performance of Panels Using Stochastic Variations of Geometric and Material Parameters," Proceedings of the 2019 AIAA Science and Technology Forum and Exposition, San Diego, CA, 2019. doi:10.2514/6.2019-0511, URL https://doi.org/10.2514/6.2019-0511 